\title{
THE GLOBULAR CLUSTER SYSTEM OF NGC 1399. I. A WIDE-FIELD PHOTOMETRIC STUDY ${ }^{1}$
}

\author{
B. Dirsch, ${ }^{2}$ T. Richtler,${ }^{2}$ D. Geisler,${ }^{2}$ J. C. Forte, ${ }^{3}$ L. P. Bassino, ${ }^{3}$ And W. P. Gieren ${ }^{2}$ \\ Received 2002 July 30; accepted 2002 December 26
}

\begin{abstract}
We present a photometric investigation of the globular cluster population of NGC 1399, the central galaxy in the Fornax cluster, in Washington $C$ and Kron $R$ filters within a field of $36^{\prime} \times 36^{\prime}$, corresponding to about $200 \times 200 \mathrm{kpc}$ at the Fornax distance. This is the largest area around this galaxy ever studied with CCD photometry. The cluster system of NGC 1399 is found to extend farther than $100 \mathrm{kpc}$ away from the galaxy. The color distribution exhibits a pronounced bimodality. Within a radial distance of about $55 \mathrm{kpc}$, the red clusters are more strongly concentrated toward the center than the blue clusters. At larger radii, the surface density profiles of the clusters are indistinguishable and match well the distribution of the galaxy light. Over the entire radial distance range, the surface brightness profile of NGC 1399 can be very well fitted by a power law with an exponent of -1.85 and a core radius of 3 ".3. No steepening of the luminosity profile can be detected at large radii. We suggest that the power-law profile of NGC 1399 results from the galaxy being embedded in a large dark matter halo, which prevents the stellar density profile from steepening outward. The cluster system contains $6450 \pm 700$ clusters and the specific frequency is found to be $5.1 \pm 1.2$ in the $V$ band. While NGC 1399 shows a pronounced color gradient the nearby comparison galaxy NGC 1404 does not show such a gradient. Using simple assumptions about the underlying population that formed during the same star formation event as the globular clusters, we present a model in which we use radially changing local specific frequencies for the red and blue subpopulations to fit the observations. We find that within $7^{\prime}$ the required specific frequency of the blue clusters alone is a factor of approximately 3 larger than that of the red ones. Outside this radius, however, both populations have the same high local specific frequency of around 8 and 13 (blue and red clusters, respectively).
\end{abstract}

Key words: galaxies: elliptical and lenticular, cD — galaxies: halos — galaxies: individual (NGC 1399) — galaxies: photometry — galaxies: star clusters

\section{INTRODUCTION}

One of the central and challenging topics in the context of the structure and evolution of early-type galaxies is the investigation of their globular cluster systems (GCSs), how they formed, and what they might tell us about the formation of their host galaxies (for recent reviews on the subject, see Ashman \& Zepf 1998; Elmegreen 1999; van den Bergh 2000; Harris 2001).

It has now emerged from numerous studies (e.g., Kundu \& Whitmore 2001a, 2001b; Larsen et al. 2001; Gebhardt \& Kissler-Patig 1999; Geisler, Lee, \& Kim 1996; Whitmore \& Schweizer 1995; Zepf \& Ashman 1993) that GCSs of early-type galaxies are by no means homogeneous populations but exhibit substructure in terms of metallicity, kinematics, and spatial distribution. The formation of massive clusters does not occur in isolation but probably needs to be accompanied by strong star-forming activity in general. This is apparent in ongoing merger events (see the list in Harris 2001) but also in the case of "normal" spiral galaxies (Larsen \& Richtler 1999, 2000). Therefore, the presence of GCs must be indicative of a corresponding field population with similar ages and metallicities, and thus GCs might be considered tracers of this field population, which otherwise is inaccessible because of the low surface

\footnotetext{
${ }^{1}$ Based partly on observations collected at the European Southern Observatory, Cerro Paranal, Chile; ESO program 66.B-0393.

2 Universidad de Concepción, Departamento de Física, Casilla 160-C, Concepción, Chile; bdirsch@cepheid.cfm.udec.cl.

${ }^{3}$ Facultad de Ciencias Astronómicas y Geofísicas, Universidad Nacional de La Plata, Paseo del Bosque S/N, 1900-La Plata, Argentina.
}

brightness of galaxy halos and/or because it is mixed up with other populations.

It might then be possible to trace back the population composition of an elliptical galaxy, the distinct metallicities and spatial distributions of the subcomponents, and compare these properties with what is expected if, for example, the elliptical galaxy is a result of a merger (or multiple mergers) of disk galaxies (Bekki et al. 2002). However, the relation of a GCS and its components to the field components can be complicated. Even if the efficiency of cluster formation per unit mass had a universal value, as argued by McLaughlin (1999), it does not mean that one can infer directly the luminosity of the underlying field population from the number of globular clusters because generally the fate of the gas is unknown.

A promising target to gain better insight into the connection between the relation of cluster subpopulations to the underlying field population is NGC 1399, the central galaxy of the Fornax cluster. It has long been known to possess a large number of globular clusters (Dawe \& Dickens 1976) out to large radii (Hanes \& Harris 1986; Harris \& Hanes 1987). Photographic studies of NGC 1399 showed that its luminosity profile cannot be described by a de Vaucouleurs profile but by a power law (Schombert 1986; Killeen \& Bicknell 1988; Caon, Capaccioli, \& D'Onofrio 1994). Whether NGC 1399 is a cD galaxy or not depends on the applied definition, which in turn differs from author to author. For example, Bridges, Hanes, \& Harris 1991 classify it as a cD, while Schombert 1986 does not include it in his list of $\mathrm{cD}$ galaxies. The spatial distribution of galaxy light and globular clusters have been compared by Bridges et al. (1991) and Wagner, Richtler, \& Hopp (1991), who, along with Geisler 
\& Forte (1990) performed the first CCD studies of the GCS of NGC 1399, and also obtained color information. Except for the very inner regions, both studies found the surface density profile of the clusters to agree well with the galaxy light. Wagner et al. and Bridges et al. also obtained the first color information of the GCS by using the $B$ and $V$ bands. While Wagner et al. could not see a color gradient, Bridges et al. detected a shift of $0.2 \mathrm{mag}$ to the blue between 0.5 and $3^{\prime}$. The latter authors, moreover, derived a turnover magnitude of $V=23.8$ and a distance of $18.5 \mathrm{Mpc}$, which still is in very good agreement with modern values (Richtler et al. 2000). The specific frequency (the total number of globular clusters normalized to the host galaxy's luminosity; Harris \& van den Bergh 1981) derived by Harris \& Hanes (1987), Bridges et al. (1991), and Wagner et al. (1991) was unusually large for an elliptical galaxy (16 \pm 4$)$, a property shared with Geisler \& Forte (1990) other central galaxies in galaxy clusters (e.g., Harris 2001).

The next contribution came from the Washington system: Ostrov, Geisler, \& Forman (1993) measured Washington colors of NGC 1399 globular clusters and also detected the color gradient reported earlier. They also mentioned a "multimodality" in the metallicity distribution, indicating that the GCS could be composed of different populations with distinct properties. Kissler-Patig et al. (1997) also noted the broad $V-I$ color distribution of the NGC 1399 clusters in comparison with other Fornax elliptical galaxies and a high specific frequency but could not confirm the color gradient seen by Ostrov et al. (1993) and Bridges et al. (1991).

An improved photometric analysis in the Washington system (Geisler \& Forte 1990, Ostrov, Forte, \& Geisler 1998) showed that the color distribution of the clusters was rather "bimodal" than "multimodal." In this work, a rather low specific frequency of only 5.6 was derived.

The open questions are still: How far does the NGC 1399 GCS extend? What substructure can be identified and how does it relate to the host galaxy? Does the population structure change in the outer parts? How does the cluster distribution compare with the stellar distribution? What significance does the quoting of a general specific frequency have if the GCS is substructured?

From the photometric side, progress can be expected only if a much larger field is investigated than has been done in the past. With the advent of the new MOSAIC cameras, we now have the opportunity to do this. Moreover, the excellent metallicity resolution of the Washington photometric system enables us to reconsider the metallicity distribution based on much better number statistics than in previous studies.

This paper is part of a larger effort to study NGC 1399 and its cluster population. The second paper in this series by Richtler et al. (2002a, hereafter Paper II) will use spectroscopic observations of globular clusters for a dynamical study of NGC 1399.

\section{OBSERVATION AND REDUCTION}

\subsection{Data and Basic Reduction}

Data from five observing runs are contributing to the present photometric study. The main data set consists of Washington wide-field images of NGC 1399 (plus a background field) taken with the MOSAIC camera mounted at the prime focus of the Cerro Tololo Inter-American Observatory (CTIO) $4 \mathrm{~m}$ Blanco telescope. Additionally, three subfields were observed at the VLT (FORS2) in $V$ and I. The location of the MOSAIC field around NGC 1399 and the VLT fields are shown in Figure 1 as overlays on a DSS image.

We used the Kron-Cousins $R$ and Washington C filters, although the genuine Washington system uses $T 1$ instead of $R$. However, Geisler (1996) has shown that the KronCousins $R$ filter is more efficient than $T 1$ because of its larger bandwidth and higher throughput and that $R$ and $T 1$ magnitudes are closely related, with only a very small color term and zero-point difference $(R-T 1 \approx-0.02)$. The MOSAIC wide-field camera images a field of $36^{\prime} \times 36^{\prime}$ at a pixel scale of 0 ".27 pixel $^{-1}$. The first three observing runs were performed in the eight-channel readout mode; for the last one (2001 November) the 16 channel mode was available. Further information on the MOSAIC camera can be found in the MOSAIC home page. ${ }^{4}$

During the first run (1999 December), we observed one MOSAIC field centered near NGC 1399 (see Fig. 1) in the $C$ filter, while the $R$ band images were kindly obtained by $\mathrm{D}$. Zurek and M. Shara. In the second run (2000 December), we observed a background field 3.5 northeast of NGC 1399 (R.A. $=3^{\mathrm{h}} 48^{\mathrm{m}}$, decl $=-32^{\circ} 32^{\prime}$ ) in $C$ and $R$, as well as Washington standard fields.

In the third run (2001 November), we observed adjacent fields to NGC 1399 that overlap with the central field. We also observed standard fields under photometric conditions. This run was used for the photometric calibration of the presented Washington data.

In addition to the MOSAIC data, we analyzed three fields $(6 ! 8 \times 6 ! 8)$ near NGC 1399 for which 5 minute exposures in $V$ and $I$ were taken with VLT/FORS2 in 2000 December, in the course of a campaign aimed at measuring radial velocities of globular clusters around NGC 1399 (Richtler et al. 2002b). In Table 1 we summarize the relevant information on the different observing runs.

The MOSAIC data were reduced using the MSCRED package within IRAF. In particular this software is able to correct for the variable pixel scale across the CCD, which would otherwise cause a $4 \%$ variability of the brightness of stellar-like objects from the center to the corners. The flatfielding resulted in images that had remaining sensitivity variations $\leq 1.5 \%$. In particular chip 4 and chip 5 showed discernible remaining flat-field structure (but within the given deviation).

To facilitate the search for point sources on the MOSAIC images, model light profiles of NGC 1399, as well as that of the neighboring galaxies NGC 1404 and NGC 1387, were subtracted. This was done using a median filter with an inner radius of $9 . .5$ and an outer radius of $11^{\prime \prime}$. This size is large enough to prevent altering the point source photometry, which has been verified with artificial-star tests described below.

\subsection{Identification of Point Sources}

We used SExtractor (Bertin \& Arnouts 1996) and DAOPHOT II for image classification and photometry, respectively. Our extensive tests showed that each program was optimized for these respective tasks. To distinguish between point sources and extended objects, we compared the SExtractor stellarity indices for objects found both on the

\footnotetext{
${ }^{4}$ See http://www.ctio.noao.edu/mosaic.
} 


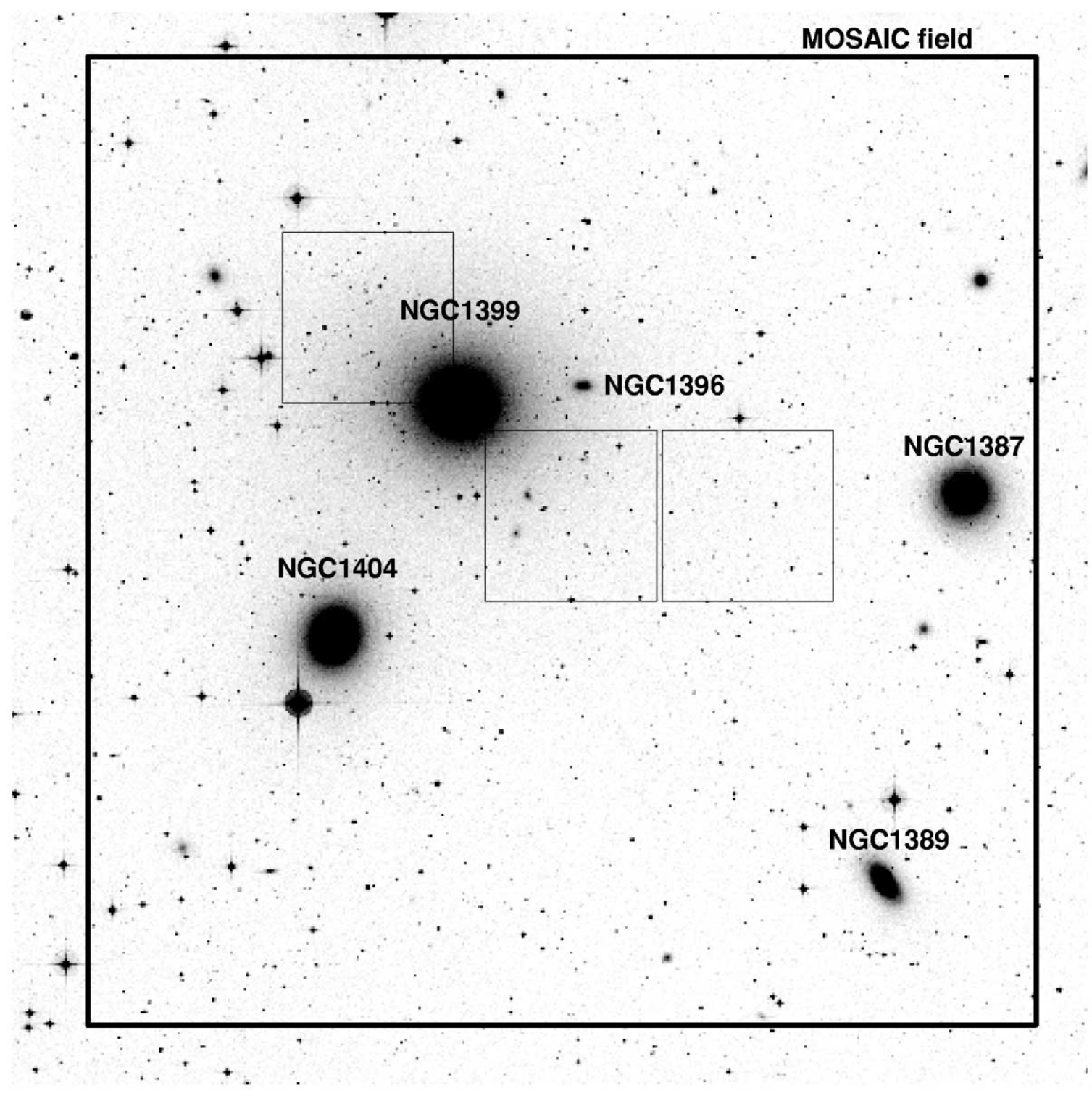

FIG. 1.-MOSAIC field and the three smaller VLT fields overlaid on a DSS image

MOSAIC and the VLT images. With the aid of the superior seeing on the VLT images, we determined 0.4 as a lower limit for the SExtractor classifier on the MOSAIC images.

Using this classification, the comparison with the VLT fields revealed that $90 \% \pm 5 \%$ of the point sources found in the MOSAIC fields were correctly classified as point sources. Only $1.2 \pm 0.1$ galaxies $\operatorname{arcmin}^{-2}$ had been erroneously classified as point sources in the MOSAIC images. With a less strict selection criterion one could reduce the number of lost point sources. However, this would come at the expense of additional contamination by background galaxies. Since we are interested in the GCS at large radii, i.e., at low surface densities, we rather prefer a lower completeness to an increased contamination by galaxies. We retained 10,453 point sources while 13,600 resolved objects were discarded. The brightest nonsaturated objects have $R \approx 18$ (slightly depending on the individual MOSAIC chip).

For further study we chose a faint-magnitude cutoff, depending on color. The limit also accounts for the fact that the background field is not as deep as the NGC 1399 field. We therefore defined this cutoff by the completeness limit of the background field and regarded only objects brighter than this limit (see Fig. 3).

\subsection{Photometric Calibration}

Standard fields for the photometric calibration have been observed during the 2001 November run. On each of the three nights, we observed four or five fields, each containing about 10 standard stars from the list of Geisler (1996), with a large coverage of air masses (typically from 1.0 to 1.9). It was possible to use a single transformation for all three nights, since the coefficients derived for the different nights were indistinguishable within the errors.

We derived the following relations between instrumental and standard magnitudes:

$$
\begin{aligned}
T 1=R_{\text {inst }}+(0.72 \pm 0.01)- & (0.08 \pm 0.01) X_{R} \\
& +(0.021 \pm 0.004)(C-T 1), \\
C=C_{\text {inst }}-(0.06 \pm 0.02)- & (0.30 \pm 0.01) X_{C} \\
+ & (0.074 \pm 0.004)(C-T 1) .
\end{aligned}
$$


TABLE 1

Observational Data Used in This Investigation

\begin{tabular}{|c|c|c|c|c|c|c|}
\hline Telescope & Date & R.A. & Decl. & Filter & $\begin{array}{l}\text { Exposure Time } \\
\text { (s) }\end{array}$ & Seeing \\
\hline MOSAIC.. & $1999 \operatorname{Dec} 7$ & 33812.0 & -353225 & $R$ & 420 & $1 " .1$ \\
\hline MOSAIC ... & 1999 Dec 7 & 33812.0 & -353225 & $R$ & 420 & $1 . " 0$ \\
\hline MOSAIC... & $1999 \operatorname{Dec} 7$ & 33812.0 & -353225 & $R$ & 420 & 0.9 \\
\hline MOSAIC.. & 1999 Dec 11 & 33812.0 & -353225 & $R$ & 420 & $1 " .2$ \\
\hline MOSAIC ................ & 1999 Dec 11 & 33812.0 & -353225 & $R$ & 420 & $1 " .2$ \\
\hline MOSAIC ................ & 1999 Dec 11 & 33812.0 & -353225 & $R$ & 420 & $1 " 3$ \\
\hline MOSAIC ............... & 1999 Dec 12 & 33812.0 & -353225 & $R$ & 420 & $1 " .2$ \\
\hline MOSAIC ................ & 1999 Dec 15 & 33812.0 & -353226 & $C$ & 1500 & $1 " ! 1$ \\
\hline MOSAIC ............... & 1999 Dec 15 & 33813.0 & -353215 & $C$ & 1500 & $1 "$ ! 1 \\
\hline MOSAIC ............... & 1999 Dec 15 & 33813.0 & -353232 & $C$ & 1500 & $1{ }^{\prime \prime} 0$ \\
\hline VLT ......................... & 2000 Dec 1 & 33845 & -352330 & $V$ & 300 & 0.55 \\
\hline VLT .......................... & 2000 Dec 1 & 33845 & -352330 & $I$ & 300 & 0.55 \\
\hline VLT ........................ & $2000 \operatorname{Dec} 2$ & 33808 & -353100 & $V$ & 300 & $0 ! 64$ \\
\hline VLT ......................... & $2000 \operatorname{Dec} 2$ & 33808 & -353100 & $I$ & 300 & $0 " 6$ \\
\hline VLT ......................... & $2000 \operatorname{Dec} 3$ & 33730 & -353130 & $V$ & 300 & 0.56 \\
\hline 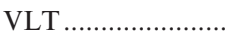 & $2000 \operatorname{Dec} 3$ & 33730 & -353130 & $I$ & 300 & 0.5 \\
\hline MOSAIC ................ & 1999 Dec 31 & 34800.0 & -353500 & $R$ & 120 & $1{ }^{\prime \prime} 0$ \\
\hline MOSAIC ................ & 1999 Dec 31 & 34800.0 & -353500 & $R$ & 600 & $1{ }^{\prime \prime} 0$ \\
\hline MOSAIC .................. & 1999 Dec 31 & 34800.0 & -353500 & $C$ & 1800 & $1{ }^{\prime \prime} 0$ \\
\hline
\end{tabular}

NotE.-Units of right ascension are hours, minutes, and seconds, and units of declination are degrees, arcminutes, and arcseconds.

The standard deviation of the difference between our calibrated and tabulated magnitudes is $0.018 \mathrm{mag}$ in $T 1$ and 0.027 mag in $C$ (see Fig. 2).

To calibrate the NGC 1399 field we identified isolated stars that were observed on three overlapping fields during the 2001 November run. These stars were used to determine the zero points, while the color terms were adopted from the 2001 November run. The scatter between the zero points determined from individual stars is 0.03 mag, most probably due to flat-field uncertainties.

The final uncertainties of the zero points are 0.03 and 0.04 mag for $R$ and $C$, respectively. This results in a calibration uncertainty in $C-T 1$ of $0.05 \mathrm{mag}$ (the uncertainty in the color term can be neglected).
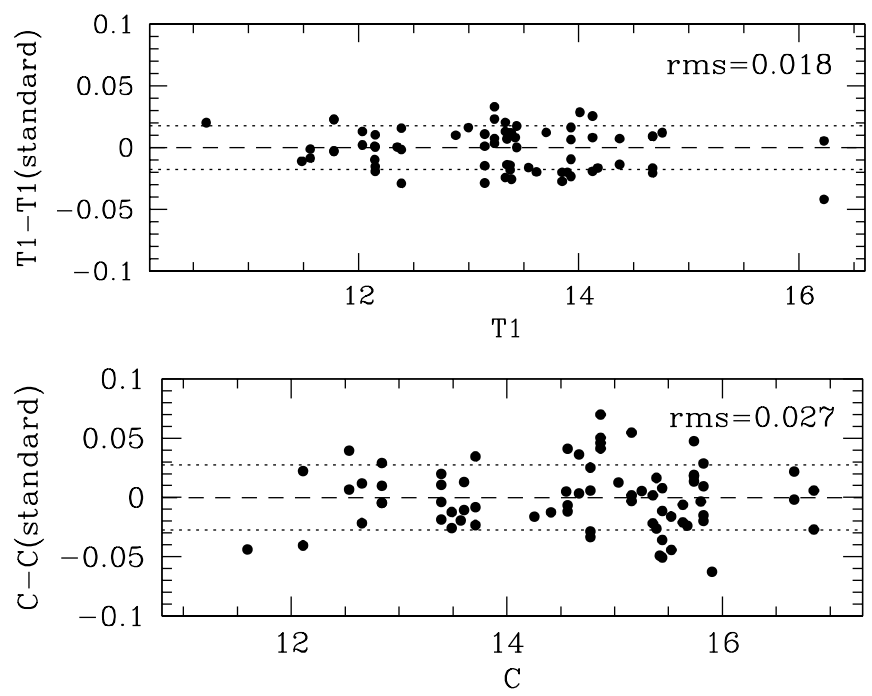

FIG. 2.- Residuals of the standard star magnitudes after plotting the applied calibrations.
Ostrov et al. (1998) also obtained Washington photometry for a smaller field centered on NGC 1399. We used their Figures 6 and 10 to obtain the color and luminosity distribution of their objects. In Figure 10 we compare the color distribution of the same subfield, and in Figure 9 the luminosity functions are compared. These figures show the good agreement between the two photometric data sets.

The VLT data in $V$ and $I$ have been calibrated using the zero points, air-mass coefficients, and color terms published on the ESO Web page for the corresponding night. ${ }^{5} \mathrm{We}$ used the same zero point for all fields despite the fact that the gain is different for all four amplifiers, since we divided by a normalized flat field and thus we had to average the gain as well (in the high-gain mode). The ESO calibration for the $I$ filter includes a color term, $I_{0} \propto I_{\text {inst }}-0.07(V-R)$. Because we had no VLT observations in the $R$ band, we used a subset of clusters for which we had VLT $(V$ and $I)$ and MOSAIC $(R)$ observations and found the following relation between $V-I$ and $V-R$ colors: $V-R=$ $-(0.10 \pm 0.04)+(0.47 \pm 0.04)(V-I)$, with a scatter of $\sigma_{V-R}=0.04 \mathrm{mag}$. This relation is used for the photometric calibration when no $R$ observations were available. This procedure introduces an error of $0.04 \mathrm{mag}$ in the final magnitudes as one can judge from the scatter of the relation. For Milky Way GCs the following can be obtained with the data assembled in the McMaster catalog (Harris 1996): $V-R=-(0.01 \pm 0.01)+(0.49 \pm 0.01)(V-I)$. Thus while the slope of the relation is the same the $V-R$ color is either 0.09 mag bluer in NGC 1399 than in the Milky Way or the $V-I$ color is systematically 0.09 mag redder. The reason for this difference is unclear. We found for the three nights

\footnotetext{
${ }^{5}$ See http://www.eso.org/observing/dfo/quality/FORS/qc/ zeropoints/zeropoints.html.
} 
(the subscripts denote the nights)

$$
\begin{aligned}
V_{1} & =v_{\text {inst }}+28.057+0.74-0.16 X_{V} V_{1}, \\
I_{1} & =i_{\text {inst }}+27.284+0.74-0.16 X_{I}-0.07\left(V_{1}-R_{1}\right), \\
V_{2} & =v_{\text {inst }}+28.048+0.74-0.16 X_{V}, \\
I_{2} & =i_{\text {inst }}+27.263+0.74-0.16 X_{I}-0.07\left(V_{2}-R_{2}\right), \\
V_{3} & =v_{\text {inst }}+28.038+0.74-0.16 X_{V}, \\
I_{3} & =i_{\text {inst }}+27.261+0.74-0.16 X_{I}-0.07\left(V_{3}-R_{3}\right) .
\end{aligned}
$$

We show the term due to the conversion from ADUs to electrons $(0.74)$ separately to facilitate the comparison with the zero points given in the ESO Web page.

For the reddening toward NGC 1399 we adopted, according to Schlegel, Finkbeiner, \& Davis (1998), $E_{B-V}=$ 0.013 . Using $E_{C-T 1}=1.97 E_{B-V}$ (Harris \& Canterna 1977) we have to correct $C-T 1$ by $0.026 \mathrm{mag}$. For the comparison field the foreground reddening is negligible: $E_{B-V}=0.002$.

The color-magnitude diagrams for the point sources in the MOSAIC images are plotted in Figure 3. The combined color-magnitude diagram for the three VLT fields is shown in Figure 4.

\subsection{Photometric Completeness}

The globular cluster luminosity function (GCLF) will be derived from the VLT images only, so the photometric incompleteness as a function of apparent magnitude of the MOSAIC images is not of much interest to our current study. However, it is interesting to know the global incom-

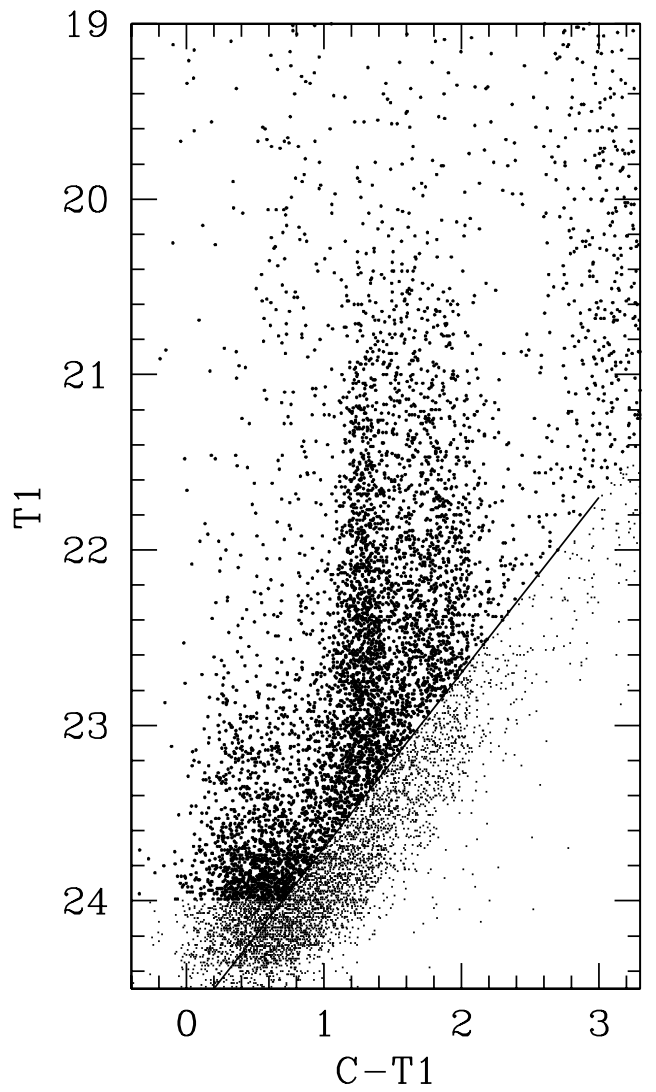

pleteness and the number of objects being resolved in the VLT images that were falsely classified as point sources in the MOSAIC images. We found that within our magnitude range $41 \% \pm 3 \%$ of the cluster candidates visible on the VLT frames were detected on the MOSAIC images and that 0.5 objects arcmin ${ }^{-2}$ resolved in the VLT images have been classified as point sources in the MOSAIC images (objects brighter than the employed faint-magnitude limit). This is a reassuringly small number.

Dithering to fill the gaps between the eight individual CCD chips was done only for the $C$ images. Therefore one has to consider the spatial incompleteness due to the gaps between the MOSAIC CCDs in the $R$ images, saturated galaxy centers, and some bright stars west of NGC 1399 that account for a spatial incompleteness of $\approx 4 \%$ in the MOSAIC data.

Experiments with artificial stars were performed to check whether a magnitude-dependent difference between input and output magnitude is observed among the point sources. This was not the case, and thus the size of the median filter we used to model and subtract the galaxy light is justified. In addition, we found that for point sources more distant from NGC 1399 than 1'6 no radially varying completeness has to be corrected for.

The completeness of the VLT fields has been determined with extensive artificial star tests. We used ADDSTAR of the DAOPHOT package under IRAF to add 5 times 2000 stars to each VLT image. The stellar point-spread function (PSF) was the same as determined by the PSF-fitting routine in the same package. The color and magnitude range

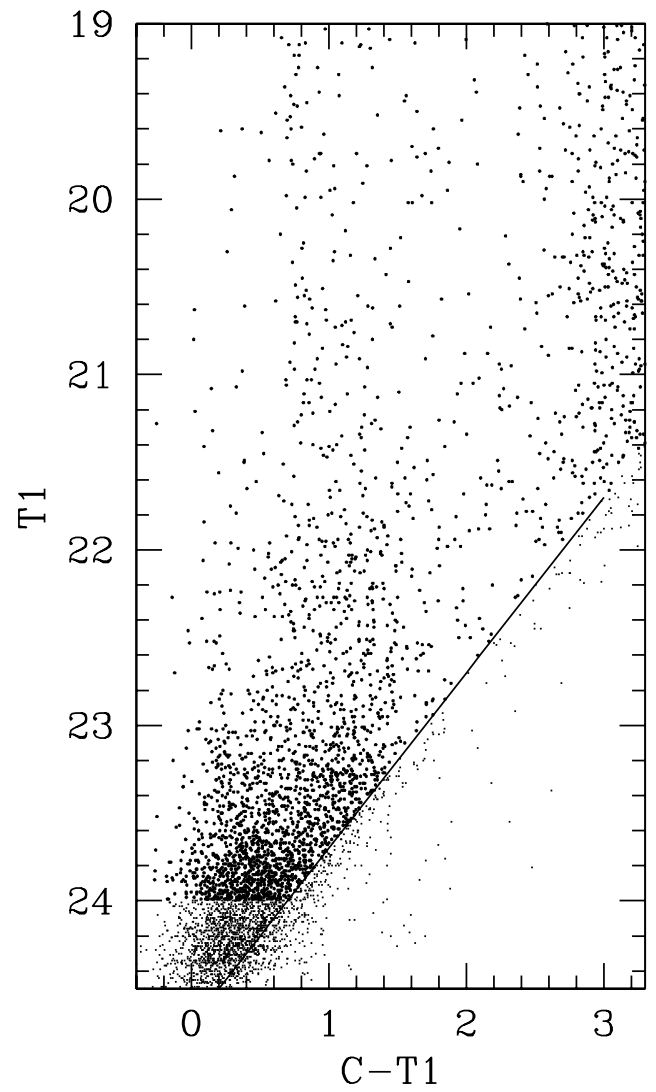

FIG. 3. - CMD based on the MOSAIC data for the NGC 1399 field (left) and the background field (right). The line indicates the limiting magnitude, which is used throughout the paper. Brighter stars are also plotted with slightly larger dots than stars fainter than the limiting magnitude. 


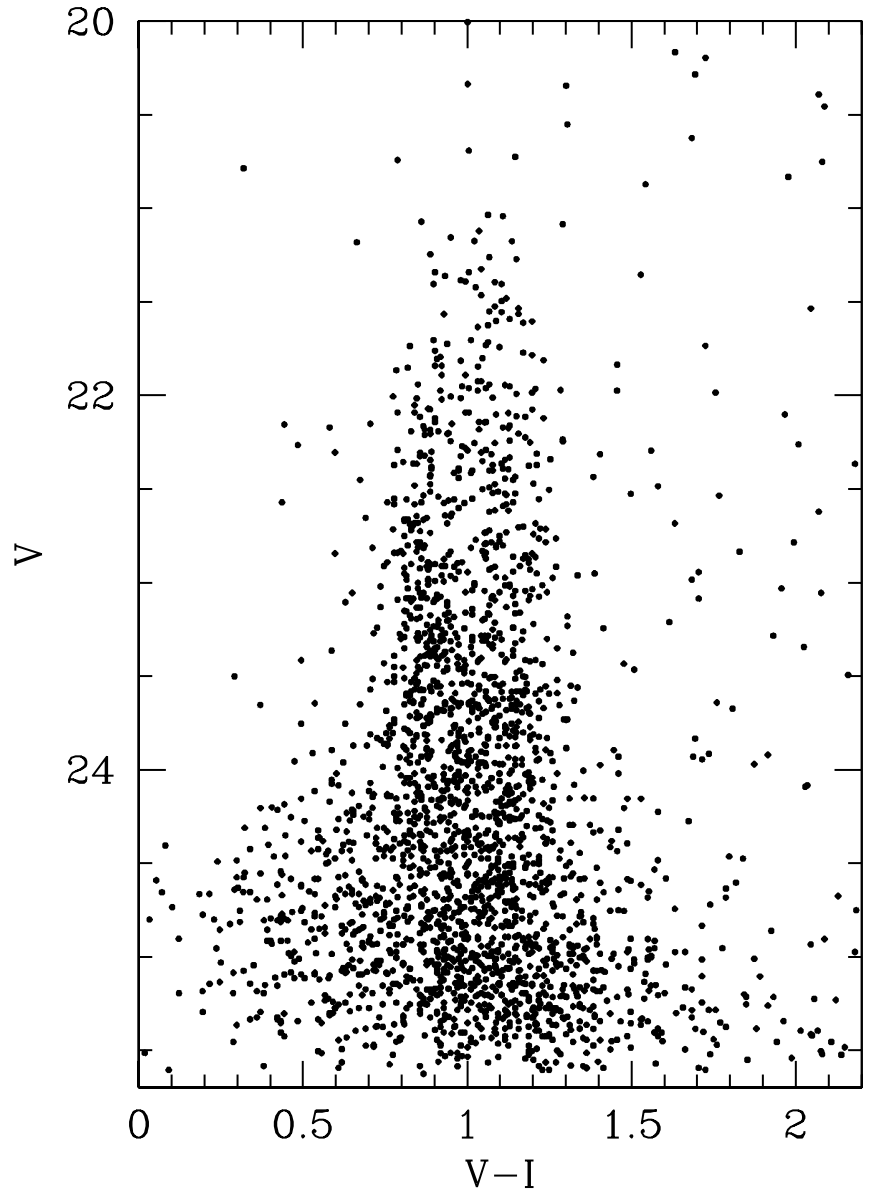

FIG. 4. - CMD assembled from the three VLT fields. The bimodality of the color distribution is visible, although not as nicely as in the Washington colors (Fig. 3). We shall use the VLT photometry mainly for deriving the globular cluster luminosity function.

encompassed the range of observed globular clusters. The resulting completeness as a function of $V$ magnitude is shown in Figure 5.

\section{COLOR-MAGNITUDE DIAGRAM}

We will now discuss the color-magnitude diagrams (CMDs) presented in Figures 3 and 4. The globular clusters show up nicely in Figure 3 within the color range

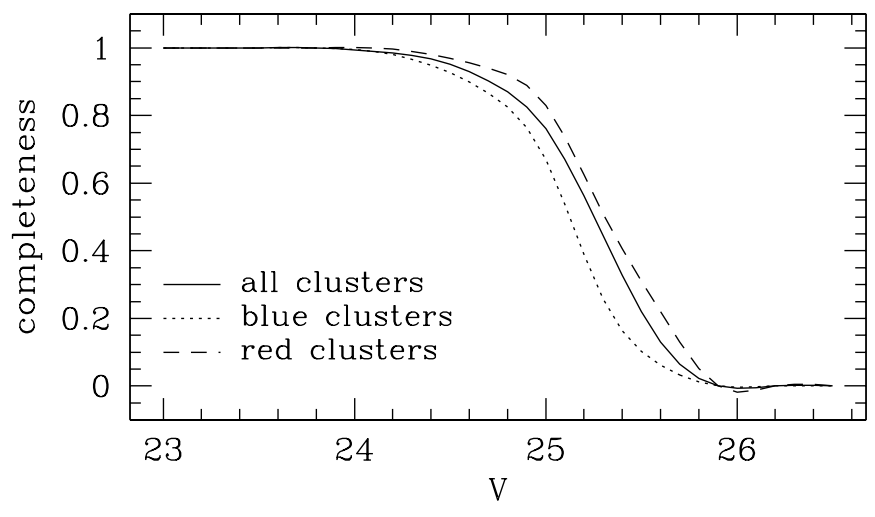

FIG. 5.-Completeness function for the VLT data (field 1)

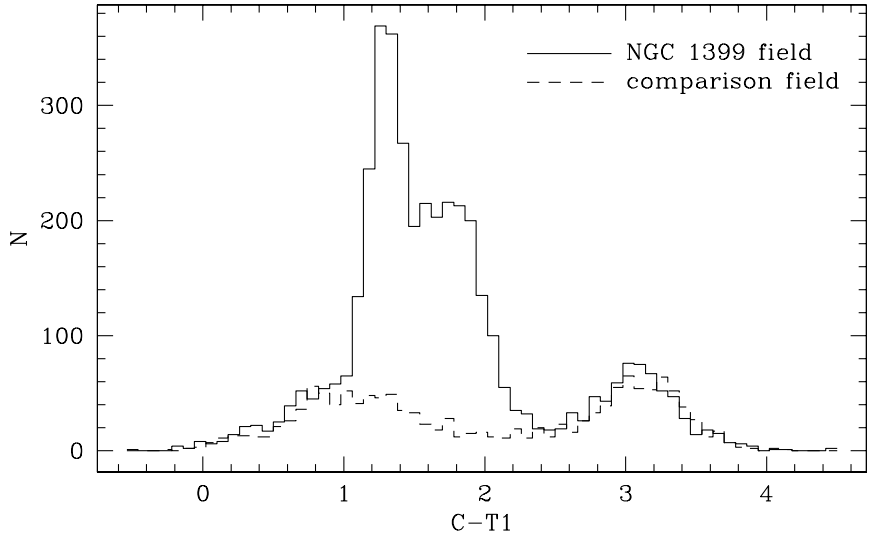

FIG. 6.-Comparison of the color histograms of all selected point sources of the NGC 1399 field (solid line) and the comparison field (dashed line).

$1.1<C-T 1<2.3$. In its vast majority the cluster population belongs to NGC 1399, but the GCSs of NGC 1404 and NGC 1387 are also included. The combination of a large area, depth, and broad color baseline render this CMD unique among the available photometric data sets of cluster systems. The cluster population starts at about $T 1=20$. Very visible is the blue "peak" in the color distribution, while the red clusters rather build a "shoulder" and do not distinctly peak at a certain color (see $\S 5$ for a detailed discussion of the color distribution). The comparison with the background field (Fig. 6) shows how well it reproduces the shoulder, starting at $C-T 1=0$ and the red peak (stars) at $C-T 1=3$.

The comparison between the VLT and the MOSAIC data also shows why the contamination of the cluster system by background galaxies is much lower for the filter combination $C$ and $R$ than for $V$ and $I$, even if the seeing is considerably worse. The reason can be seen in Figure 7. The main sources that contaminate the cluster sample in $V$ and $I$ are unresolved faint blue galaxies that have a UV excess and are thus bluer than cluster candidates in $C-R$ while having the same $V-I$ color. We confirm the galaxy nature of these $C-T 1$ blue objects since they have a homogeneous distribution and are not concentrated toward NGC 1399 like the cluster candidates.

\section{GLOBULAR CLUSTER LUMINOSITY FUNCTION}

To derive the globular cluster luminosity function (GCLF) of the VLT data we selected clusters within a radial distance of $4 ! 3$ from the center of NGC 1399. As a background field we used the FORS2 image centered $13^{\prime}$ away from NGC 1399. We are aware of the fact that this "background field" still contains a considerable number of clusters apart from background objects, which deteriorates the statistics but should not alter the location of the turnover magnitude (TOM).

The luminosity function is also plotted as kernel filtered density distributions. We followed here the approach described by Merritt \& Tremblay (1994) and used an adaptive Epanechikov kernel. The scaling in Figure 8 is the number of clusters per $0.2 \mathrm{mag}$. In the following, all distributions using this nonparametric estimation are based on the Epanechikov kernel. For the fitting we use both the filtered data and the histograms to ensure that the results do not depend on the representation. 

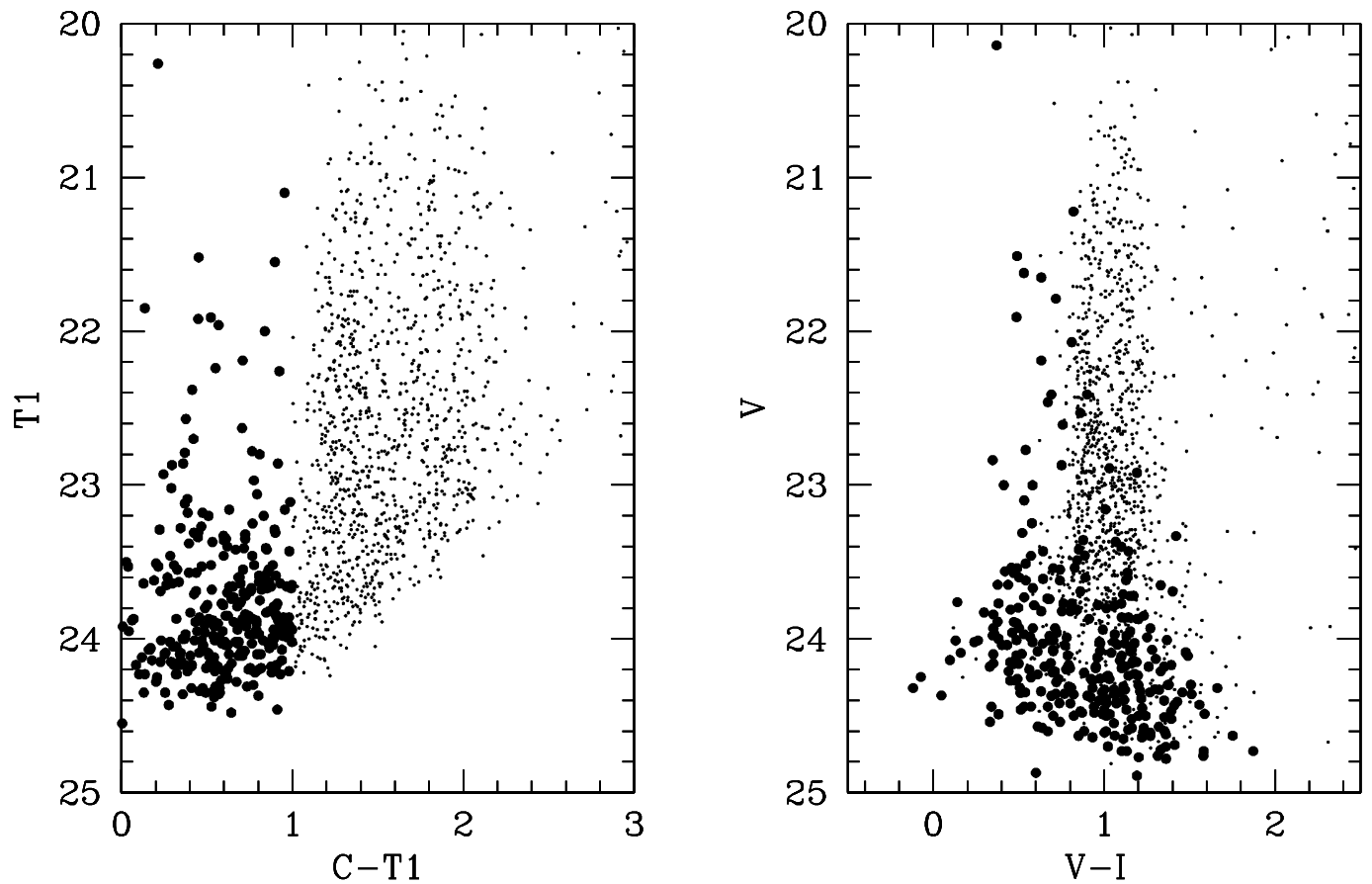

FIG. 7.-CMDs of point sources that have been identified on both the VLT and the MOSAIC images. We marked objects bluer than $C-T 1=1$ with large circles. These are predominantly galaxies. Note how these objects contaminate the CMD in $V$ and $V-I$.

We fitted a Gaussian to the distributions for different limiting magnitudes (always brighter than the 50\% completeness) and found an average $\sigma=1.30 \pm 0.12$ and $m_{\mathrm{TO}}=24.0 \pm 0.1$. However, a correlation between $\sigma$ and TOM exists in these fits because our observations do not extend far enough beyond the TOM. It is therefore advisable to keep the width fixed and to fit only the TOM. With $\sigma=1.2,1.3$, and 1.4 , which are used for elliptical galaxies (Ashman \& Zepf 1998), we find TOM of $23.98 \pm 0.11$, $24.02 \pm 0.09$, and $24.05 \pm 0.12$, respectively. We choose the same final TOM of $m_{\mathrm{TO}}=24.0 \pm 0.1$. With the knowledge

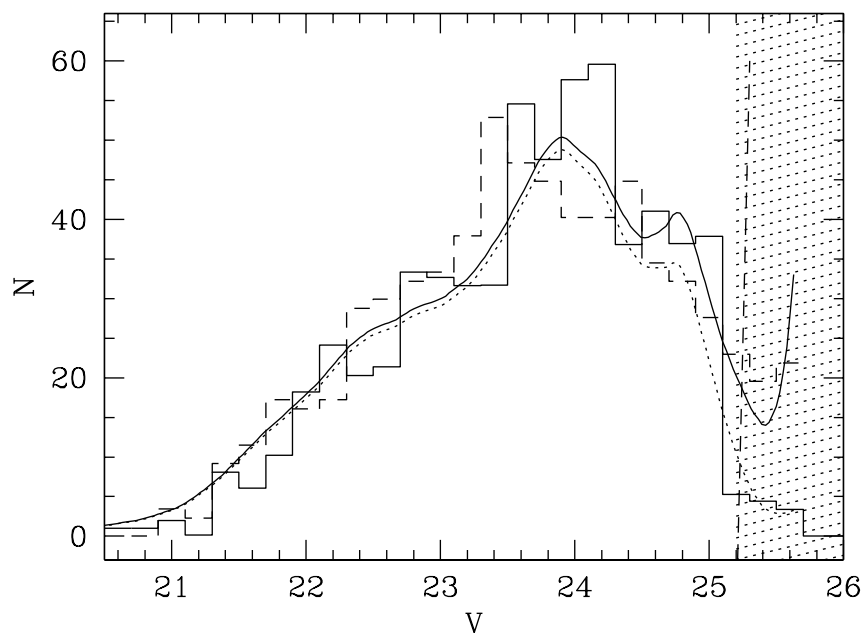

FIG. 8.-Luminosity function of the cluster candidates within a radial range of 4.3 in the FORS2 images plotted as a solid line. The shaded area designates the magnitude range beyond the $50 \%$ completeness. The dotted line shows the histogram before the completeness correction. The dashed histogram indicates the (arbitrarily scaled) HST GC luminosity function of Grillmair et al. (1999). of the TOM we calculate that the fraction of all clusters found in the area covered by the VLT data is $85 \% \pm 4 \%$.

The background-subtracted luminosity functions of the MOSAIC cluster candidates from $2^{\prime}$ to $16^{\prime}$ (excluding $2^{\prime}$ around NGC 1404) are shown in Figure 9. The luminosity distributions are consistent with the assumed distance modulus of $m-M=31.4$ and a width of the Gaussian luminosity function of 1.2 .

This TOM, together with the known completeness of the VLT data, can be used to determine the completeness of the MOSAIC fields. Since $41 \% \pm 3 \%$ of the VLT candidates were found on the MOSAIC images (see above) the absolute completeness is $36 \% \pm 5 \%$ for the MOSAIC images.

An alternative approach to find the completeness is to use the luminosity function of the MOSAIC clusters directly: using a TOM of $T 1=23.3$ and a width of $\sigma=1.2$ (Forte

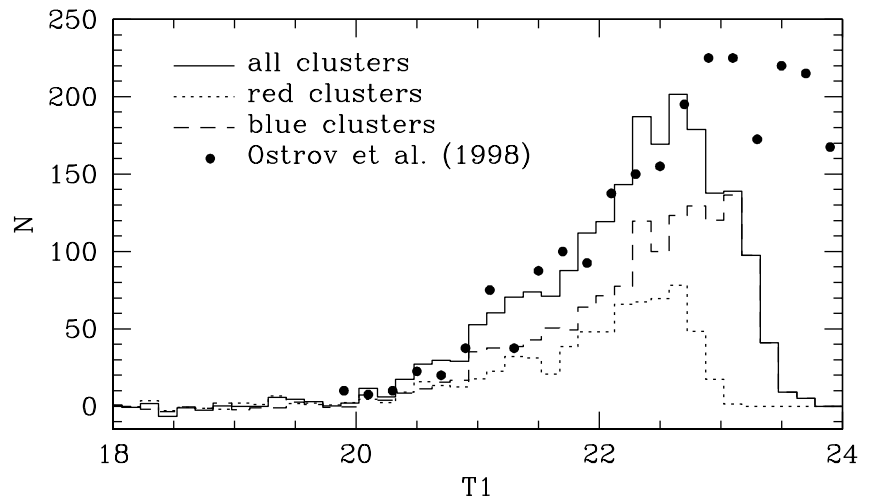

FIG. 9.-Luminosity function of the cluster candidates from the MOSAIC data for the whole sample (solid line) and for the red (dotted line) and blue (dashed line) subsamples. The luminosity function given by Ostrov, Forte, \& Geisler (1998) is also shown (arbitrarily scaled). 
et al. 2001) we find a completeness of $41 \% \pm 2 \%$. We adopt a final completeness of $34 \% \pm 5 \%$, taking into account a spatial incompleteness due to the undithered gaps of $4 \%$.

The GCLF is frequently used as a distance indicator for early-type galaxies because of its universal peak luminosity (e.g., Harris et al. 1991, Harris 2001; Ashman, Conti, \& Zepf 1995; Kundu \& Whitmore 2001a; Larsen et al. 2001). However, because of the uncertainty of the VLT calibration we do not use the obtained TOM for more than the above presented completeness calculations for which the absolute calibration is not critical.

In the following we use a distance to NGC 1399 of $m-M=31.4 \pm 0.2$, consistent with SBF distances (Tonry et al. 2001; Liu, Graham, \& Charlot 2002) but only marginally consistent with the result of Grillmair et al. (1999) from the GCLF technique.

\section{COLOR DISTRIBUTION OF THE CLUSTERS}

In the CMD of all point sources around NGC 1399 (Fig. 3) the GC candidates stand out strikingly. A blue peak at $C-T 1=1.3$ followed by a broad distribution toward redder colors is prominently visible. In the following, we define GC candidates by the magnitude interval $20<T 1<$ 23 and the color interval $0.8<C-T 1<2.3$.

In Figure 10 we plot the background-subtracted color distribution for the cluster candidates for the entire sample and in three different ranges in radial distance. It is apparent that the color distribution is radially dependent: at larger radii the relative contribution of the red clusters is much smaller than at lower radii. This already shows that the blue clusters exhibit a shallower density profile than the red clusters, as has already been found by several authors. However, it is also apparent that this might not hold for larger radii: the two outer distributions (Fig. 10, bottom left and bottom right) look quite similar and are statistically not distinguishable. The distributions plotted in Figure 10 are tabulated in Table 2. The two peaks in the innermost sample are at $C-T 1=1.32 \pm 0.05$ and $1.79 \pm 0.03$, respectively.

As mentioned above, NGC 1399 has already been studied by Ostrov et al. (1998), who found a bimodal distribution with peaks at $C-\mathrm{T} 1 \approx 1.4$ and $C-T 1 \approx 1.8$, agreeing with
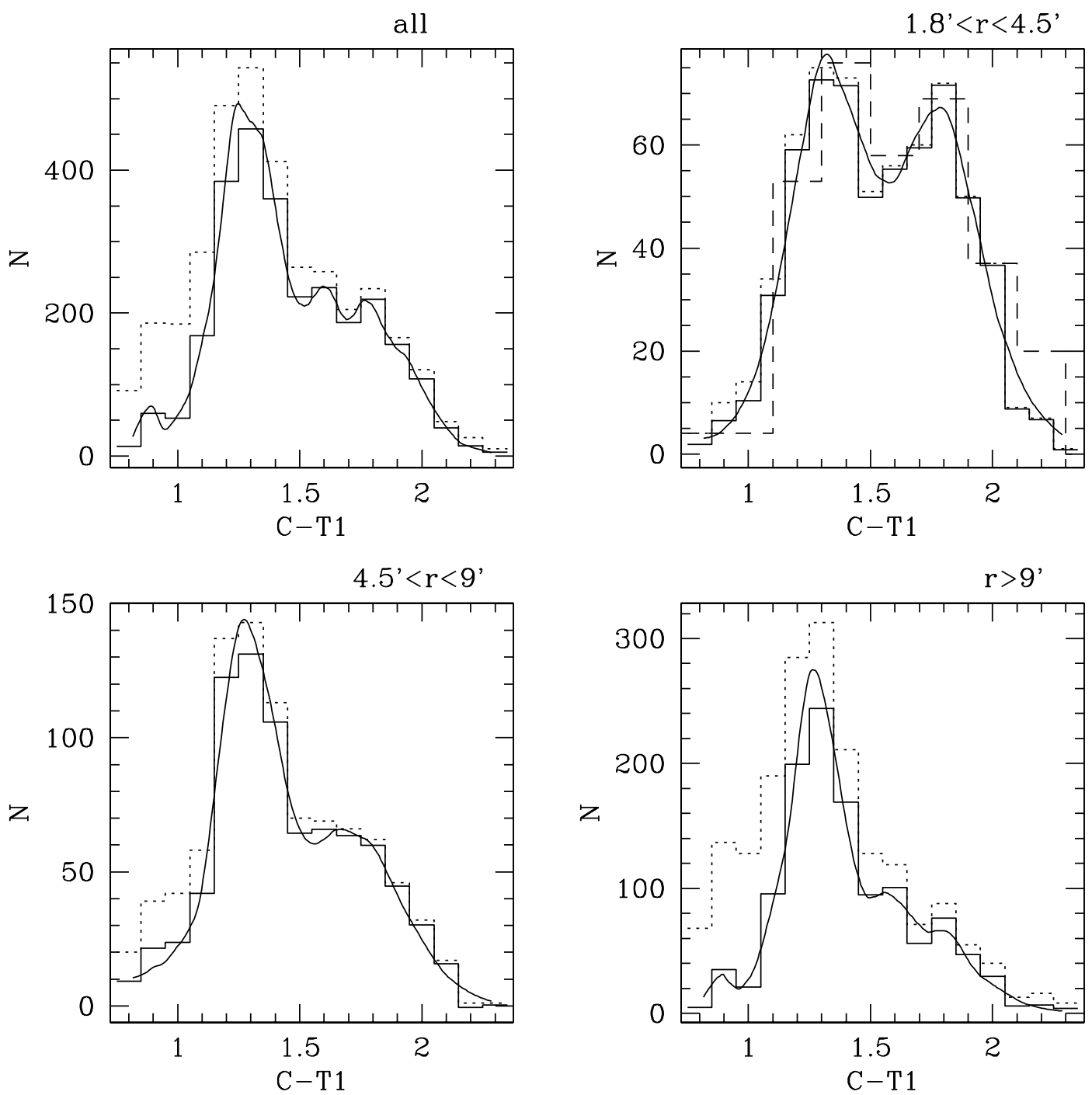

FIG. 10.-Color distribution of globular clusters for the entire sample and for three samples within different radial intervals as indicated at upper right in each panel. The dotted histogram shows the distribution before the statistical background subtraction. The smooth line is the adaptive kernel filtered distribution (described in $\S 4$ ). The dashed histogram at top right shows the color distribution by Ostrov et al. (1998) within the same area and demonstrates the good agreement of the photometry. The counts of Ostrov et al. have not been scaled. For an interpretation of these observed distributions, see $\S 5$ of the text. 
TABLE 2

Color Distribution of Clusters for the Sample and Subsamples

\begin{tabular}{crrrrr}
\hline \hline$C-R$ & \multicolumn{1}{c}{$N_{\text {all }}$} & \multicolumn{1}{c}{$N_{1}$} & \multicolumn{1}{c}{$N_{2}$} & \multicolumn{1}{c}{$N_{3}$} & \multicolumn{1}{c}{$N_{\text {BG }}$} \\
\hline $0.8 \ldots \ldots$ & $14 \pm 13$ & $2 \pm 2$ & $10 \pm 7$ & $4 \pm 8$ & $80 \pm 9$ \\
$0.9 \ldots \ldots$. & $60 \pm 18$ & $6 \pm 3$ & $31 \pm 9$ & $26 \pm 11$ & $129 \pm 11$ \\
$1 \ldots \ldots$. & $53 \pm 18$ & $10 \pm 4$ & $41 \pm 11$ & $3 \pm 11$ & $135 \pm 12$ \\
$1.1 \ldots \ldots$. & $169 \pm 20$ & $31 \pm 6$ & $96 \pm 13$ & $42 \pm 12$ & $119 \pm 11$ \\
$1.2 \ldots \ldots$. & $384 \pm 24$ & $59 \pm 8$ & $203 \pm 16$ & $119 \pm 15$ & $108 \pm 10$ \\
$1.3 \ldots \ldots$. & $458 \pm 25$ & $73 \pm 9$ & $253 \pm 17$ & $123 \pm 14$ & $87 \pm 9$ \\
$1.4 \ldots \ldots$. & $360 \pm 22$ & $72 \pm 9$ & $187 \pm 15$ & $88 \pm 12$ & $53 \pm 7$ \\
$1.5 \ldots \ldots$. & $223 \pm 17$ & $50 \pm 7$ & $109 \pm 11$ & $50 \pm 9$ & $42 \pm 6$ \\
$1.6 \ldots \ldots$. & $236 \pm 17$ & $55 \pm 7$ & $111 \pm 11$ & $55 \pm 9$ & $23 \pm 5$ \\
$1.7 \ldots \ldots$. & $186 \pm 15$ & $59 \pm 8$ & $83 \pm 10$ & $37 \pm 7$ & $19 \pm 4$ \\
$1.8 \ldots \ldots$. & $219 \pm 16$ & $72 \pm 8$ & $95 \pm 10$ & $41 \pm 7$ & $15 \pm 4$ \\
$1.9 \ldots \ldots$. & $156 \pm 13$ & $50 \pm 7$ & $68 \pm 9$ & $24 \pm 6$ & $10 \pm 3$ \\
$2 \ldots \ldots \ldots$. & $108 \pm 12$ & $37 \pm 6$ & $45 \pm 7$ & $15 \pm 5$ & $13 \pm 4$ \\
$2.1 \ldots \ldots$. & $39 \pm 8$ & $9 \pm 3$ & $18 \pm 5$ & $4 \pm 3$ & $9 \pm 3$ \\
$2.2 \ldots \ldots$. & $14 \pm 6$ & $7 \pm 2$ & $1 \pm 3$ & $4 \pm 4$ & $12 \pm 3$ \\
$2.3 \ldots \ldots$. & $5 \pm 4$ & $1 \pm 1$ & $3 \pm 2$ & $1 \pm 1$ & $5 \pm 2$ \\
\hline
\end{tabular}

Note. $-N_{\text {all }}$ is the whole sample; $N_{1}$ is the subsample within $1 ! 8<r<4 ! 5 ; N_{2}$ is the subsample within $4.5<r<13.5 ; N_{3}$ is the subsample with $r>13.5$.

our values for the inner region. This is also shown in Figure 10 (top right).

\section{RADIAL DISTRIBUTION OF THE CLUSTERS}

The area we cover is the largest ever observed for the NGC 1399 GCS. However, the innermost region is inaccessible because of the brightness of the galaxy and the saturated parts of the images. Therefore we combined our data with Hubble Space Telescope (HST) observations by Forbes et al. (1998) to include also the inner part of NGC 1399 in our study. We extrapolated all three samples (HST, VLT, and MOSAIC) to the entire luminosity function with the parameters given in $\S 4$. In Figure 11 the resulting surface densities of GCs are plotted for the three samples. The HST results are in excellent agreement with our data at overlapping radii, demonstrating the validity of our completeness

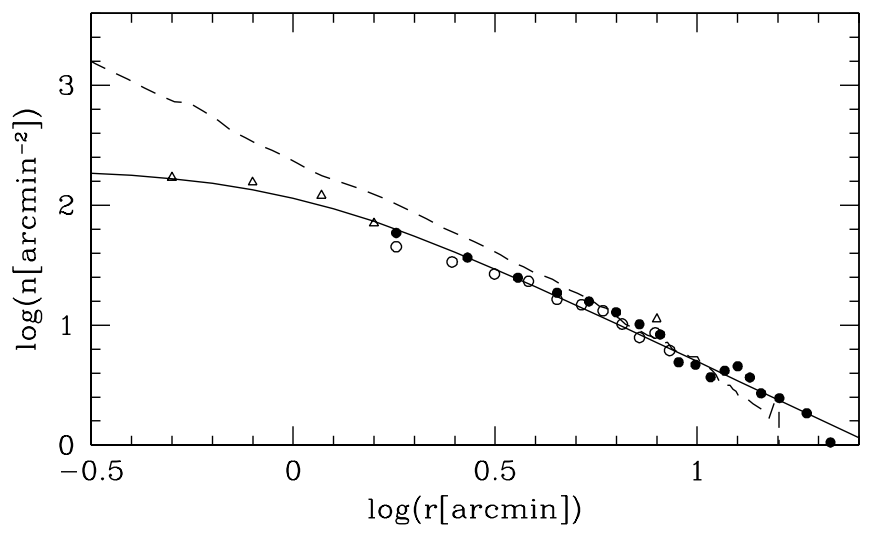

FIG. 11.- Radial density distribution of all clusters (extrapolated to the entire luminosity function) derived from the MOSAIC data (open circles), the VLT data (filled circles), and the HST data (triangles; Forbes et al. 1998). The solid line is the fit to the data. The dashed line shows the luminosity profile of NGC 1399 , which is described in $\S 7$. calculations. A fit that satisfactorily reproduces the surface densities of the total number of clusters after completeness correction is

$$
n(r)=200\left[1+\left(\frac{r}{1^{\prime}}\right)^{2}\right]^{-0.8},
$$

where $r$ is per square arcminute. In the following, we focus on the MOSAIC sample. From the color histograms in Figure 10 one can immediately see that the red clusters are more centrally concentrated than the blue ones. Guided by Figure 10 , we define the color limit between red and blue clusters to be $C-T 1=1.55$, which is the minimum seen in the clearly bimodal color distribution seen in Figure 10 (top right). We can now quantify the radial surface density distributions displayed in Figure 12, which show the density profiles for both populations (see Table 3). Because the inner region, where a core is visible, is excluded, we can describe the density profiles by pure power laws.

Out to a galactocentric radius of about $7^{\prime}$, the blue clusters show a distinctly shallower slope than the red clusters. This has already been found in earlier studies of NGC 1399 (e.g., Forbes et al. 1998; Ostrov et al. 1998), as well as in other galaxies, NGC 4636 being the most striking example (Kissler et al. 1994).

The region beyond $7^{\prime}$ has as yet been unexplored and indeed we see an interesting change. The distribution of the red population is well represented by a single power law over the full radius interval between $2^{\prime}$ and $20^{\prime}$, but the blue population shows a change from a shallow profile in the inner region to a steeper profile in the outer region. Here, two power laws are more appropriate. For the blue clusters, the profiles are proportional to $r^{-0.8 \pm 0.17}$ and $r^{-1.71 \pm 0.21}$ (for 1 ' $5<r<7^{\prime}$ and $7^{\prime}<r<21^{\prime}$, respectively). For the red population, we find the profile $\propto r^{-1.64 \pm 0.10}$. Thus we cannot see any significant difference in the slope between red and blue cluster distributions beyond $7^{\prime}$ of radial distance. This can also be quantified with a K-S test, for which we selected only clusters brighter than $R=22.5$ to minimize the effect of the background. This test results in a $48 \%$ probability that the red and blue clusters at large radii have a different

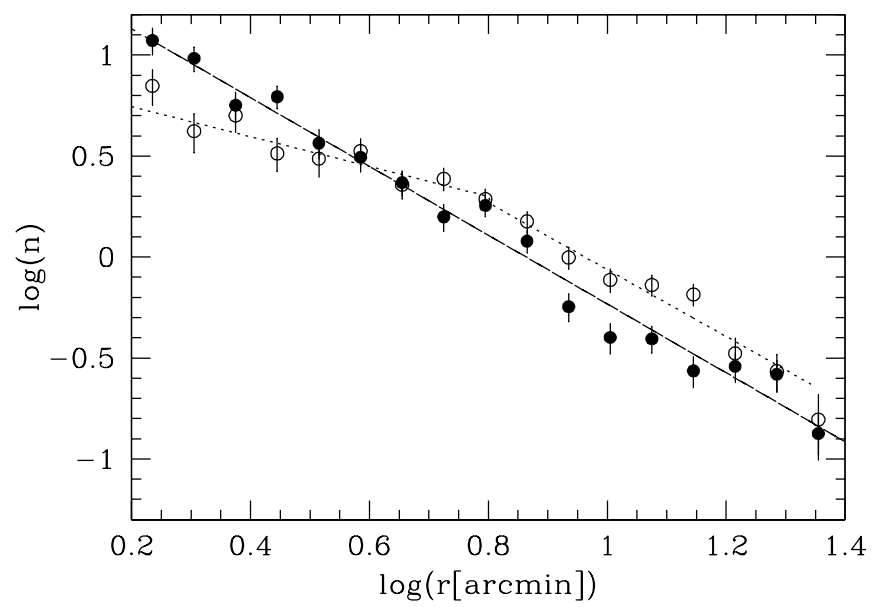

FIG. 12.-Radial density profiles of the observed number of red (filled circles) and blue (open circles) globular clusters detected on the MOSAIC image, plotted together with the fitted power laws. The counts are tabulated in Table 3. 
TABLE 3

Observed Radial Number Density of Red $[n($ RED $)]$ AND Blue $[n$ (BLUE) $]$ CluUSters

\begin{tabular}{|c|c|c|}
\hline $\begin{array}{c}\log r \\
(r \text { in arcmin })\end{array}$ & $\begin{array}{l}\log [n(\text { red })] \\
\left(\operatorname{arcmin}^{-2}\right)\end{array}$ & $\begin{array}{l}\log [n \text { (blue) }] \\
\left(\operatorname{arcmin}^{-2}\right)\end{array}$ \\
\hline $0.24 \ldots$ & $1.13 \pm 0.06$ & $0.61 \pm 0.10$ \\
\hline $0.31 \ldots \ldots$ & $1.04 \pm 0.05$ & $0.54 \pm 0.09$ \\
\hline 0.38. & $0.82 \pm 0.06$ & $0.56 \pm 0.08$ \\
\hline $0.45 .$. & $0.89 \pm 0.05$ & $0.39 \pm 0.09$ \\
\hline 0.52. & $0.69 \pm 0.06$ & $0.26 \pm 0.10$ \\
\hline 0.59. & $0.58 \pm 0.06$ & $0.40 \pm 0.07$ \\
\hline $0.66 \ldots \ldots \ldots \ldots \ldots$ & $0.46 \pm 0.05$ & $0.24 \pm 0.07$ \\
\hline $0.73 \ldots \ldots \ldots \ldots \ldots$ & $0.33 \pm 0.05$ & $0.28 \pm 0.06$ \\
\hline $0.80 \ldots \ldots \ldots \ldots \ldots$ & $0.32 \pm 0.05$ & $0.14 \pm 0.06$ \\
\hline 0.87 . & $0.20 \pm 0.05$ & $0.10 \pm 0.05$ \\
\hline $0.94 .$. & $-0.13 \pm 0.06$ & $-0.08 \pm 0.06$ \\
\hline $1.01 \ldots \ldots \ldots \ldots \ldots$ & $-0.30 \pm 0.06$ & $-0.23 \pm 0.06$ \\
\hline $1.08 \ldots$ & $-0.26 \pm 0.06$ & $-0.19 \pm 0.05$ \\
\hline $1.15 \ldots \ldots \ldots \ldots \ldots$ & $-0.42 \pm 0.06$ & $-0.24 \pm 0.05$ \\
\hline 1.22 . & $-0.43 \pm 0.06$ & $-0.57 \pm 0.08$ \\
\hline $1.29 .$. & $-0.45 \pm 0.06$ & $-0.72 \pm 0.10$ \\
\hline $1.36 \ldots \ldots \ldots \ldots \ldots$ & $-0.75 \pm 0.10$ & $-0.88 \pm 0.13$ \\
\hline
\end{tabular}

radial distribution. This probability should be considered an upper limit, since we did not correct for the background contamination, which affects the red and blue color range differently. This indicates again that the distribution of red and blue clusters is consistent with being the same.

One might recognize that the slopes fitted to the MOSAIC data at large radii are shallower than the fit to all GCs over the whole radial range presented above. This is also apparent from Figure 11, which suggests that the assumption of a uniform power law over the whole galactocentric distance range might not be adequate. At larger radii, where the investigation is restricted to the MOSAIC data, the slope is shallower.

To provide an independent check, we derived the radial profiles for the red and blue subsamples between $1^{\prime}$ and $8^{\prime}$ also from the VLT data. We used only cluster candidates brighter than $V=22 \mathrm{mag}$ to avoid the background contamination coming from the faint blue galaxies, which in $V-I$ cannot be distinguished from cluster candidates (see Fig. 7). Moreover, the VLT fields cover only approximately $11 \%$ of the MOSAIC field and thus the statistics are poorer and the errors larger. In spite of this, the resulting density distributions of metal-poor $(0.7<V-I<0.95)$ and metal-rich clusters $(1.05<V-I<1.2)$ agree very well with the results obtained with the Washington observations: The exponents are $-1.0 \pm 0.13$ and $-1.5 \pm 0.18$ for the blue and the red populations, respectively. However, a difference emerges for the blue population at small radii. If we consider only the blue clusters within $5^{\prime}$, we obtain an exponent of $-0.5 \pm 0.09$ compared with $-1.2 \pm 0.2$ (based on four points) outside this radius. This indicates that the assumption of two power laws describing the metal-poor population is probably too simple.

Besides NGC 1399, NGC 4472, which is the brightest galaxy in the Virgo Cluster, is the only elliptical galaxy in which the GCS has been studied with wide-field CCD photometry (Rhode \& Zepf 2001). Because of its similar distance (17 $\mathrm{Mpc}$ ) and similar size the results for this galaxy can be readily compared with ours. In particular the radial distributions of red and blue clusters found by Rhode \& Zepf (2001) is very similar to our results for NGC 1399. This includes the mentioned "levelling out" of the number ratio of red versus blue clusters at large radii; i.e., the same radial decline of red and blue clusters is indicated.

\subsection{Brightest Clusters}

In Figure 13 we show the color distribution for three different brightness bins. It is striking that for the brightest clusters, shown in Figure 13 (top), the color distribution differs largely from the fainter samples seen (this is already apparent in the CMD): compared with the fainter sample more intermediate colors $(C-T 1 \approx 1.55)$ are present. A Kolmogorov-Smirnov test (K-S test) resulted in only a $5 \%$ probability that the bright sample has the same color distribution as the fainter one $(R=21-22)$. Ostrov et al. (1998) suggested that the brightest clusters might have a unimodal distribution; however, their lower number of objects did not permit a clear statement. In the faintest-magnitude bin, the red peak is shifted toward the blue with respect to the intermediate bin. This, however, may be easily explained by the inclined completeness line that affects only this bin. These bright clusters are as concentrated as the red clusters and do not have the shallow distribution of the blue ones: a $\mathrm{K}-\mathrm{S}$ test results in a $1 \%$ probability that the bright ones are not distributed as the red ones and a $95 \%$ probability that they have a different distribution than the blue ones.

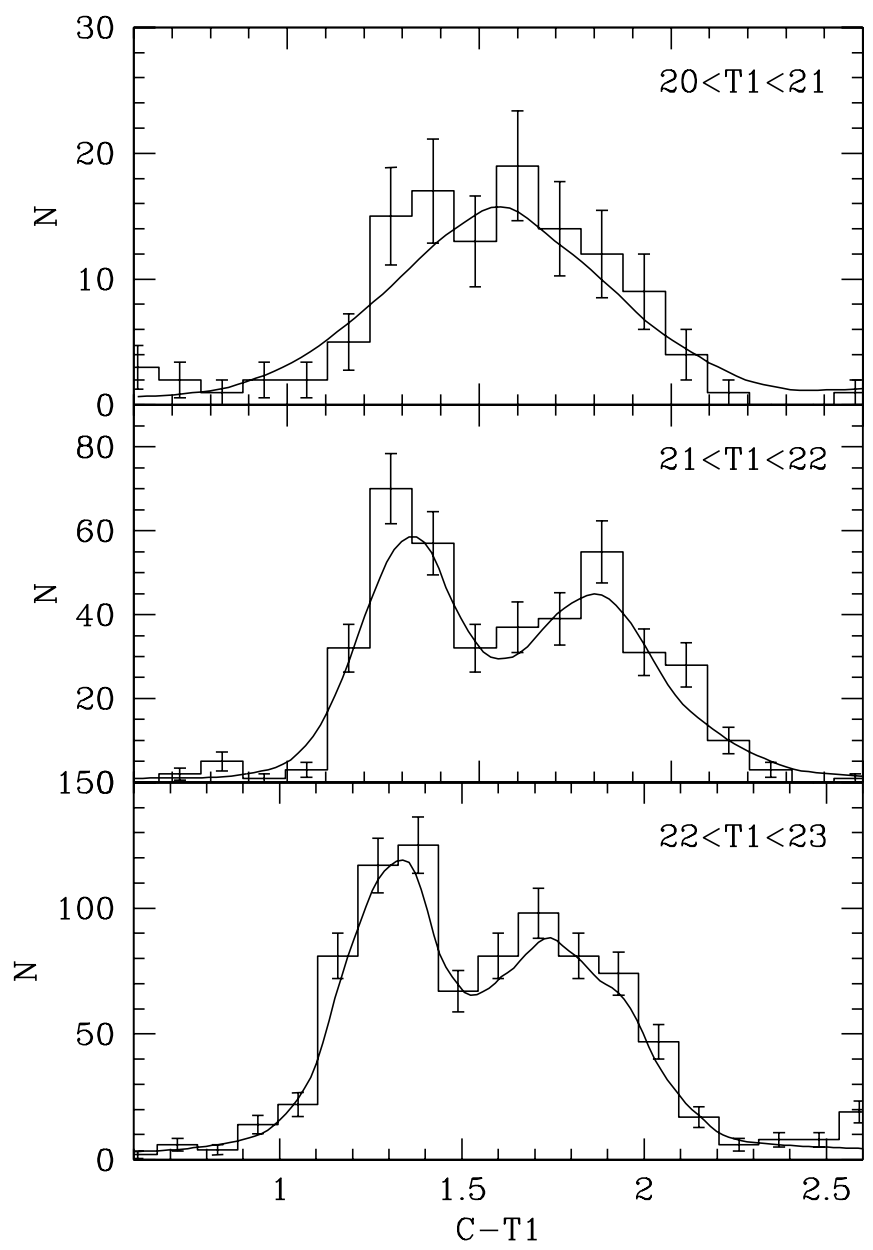

FIG. 13.-Color distribution of globular clusters for three different luminosity intervals. The brightest clusters do not exhibit a bimodal pattern. 


\section{GALAXY LIGHT PROFILE}

It is now of interest to compare the surface density profile of the GCS with that of the galaxy light and to search for any color gradient, which would indicate a correspondence between the GCS and the galaxy population itself. Previous photographic work covering a similarly large field has been carried out by Schombert (1986; $V$ band) and Caon et al. (1994; $B$ band). The resulting profiles are plotted in Figure 14. Beyond a radius of $5^{\prime}$ they begin to diverge in the sense that the $V$ profile gets brighter than the $B$ profile. Besides the fact that this "color gradient" has the very unexpected sense of getting redder, it also reaches the implausibly high value of almost 2 mag at 14'. We conclude that $V$ and/or $B$ are not reliable at faint surface brightness values. We therefore tried to derive the light profile from our images as well.

We measured the brightness around all point sources within an annulus between $8^{\prime \prime}$ and $11^{\prime \prime}$ on the point sourcesubtracted $C$ and $R$ images. We defined this brightness to be the mode of the distribution of pixel intensities after a $5 \%$ clipping of the brightest peaks. Without the $\sigma$ clipping the scatter would have been significantly larger.

Care has to be taken because of the eight different chips used and the corresponding differences in the sensitivities that are left after flat-fielding. To account for these differences we shifted the zero points of individual chips to a common mean value by adjusting the sky brightness in adjacent fields. The resulting correction factors are of the order of $0.3 \%$ of the background level in the $R$ image and of the order of $1 \%$ in the $C$ images. Since the $R$ images are deeper we used them to trace the galaxy light to large radii, whereas the $C$ data have been used to search for a color gradient at smaller
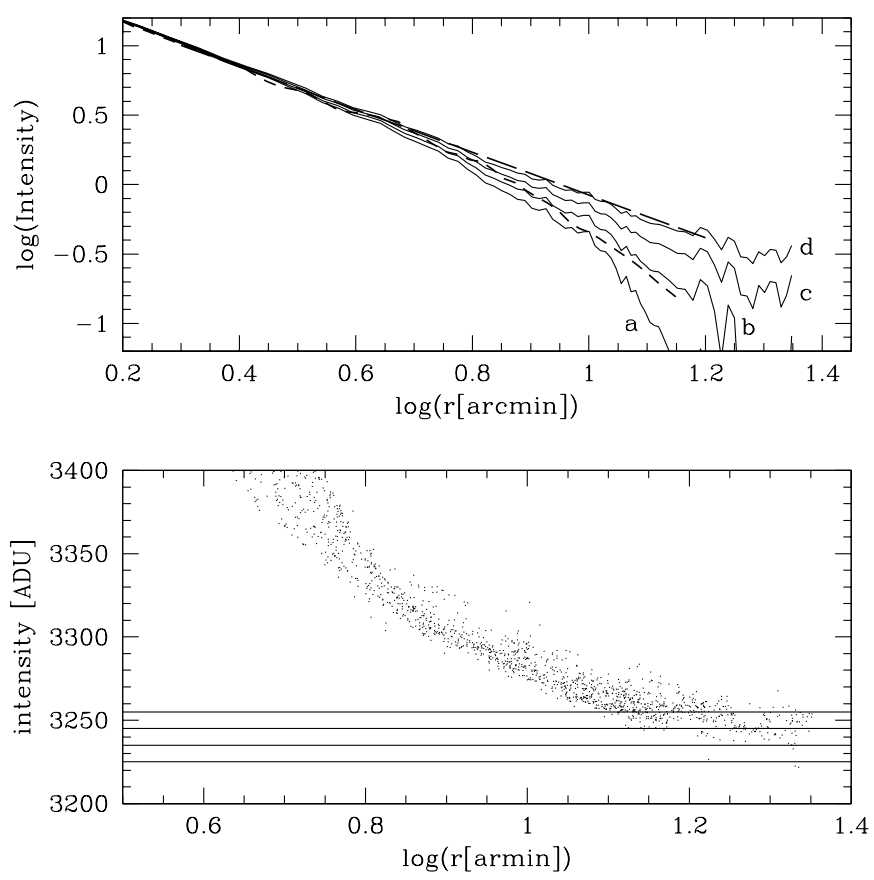

FIG. 14.-Top: Luminosity profile from our $R$ images (solid lines; for four different background values), the one published by Schombert et al. (1986; long-dashed line) and the one by Caon, Capaccioli, \& D'Onofrio (1994; short-dashed line). We shifted all profiles to the $B$ surface magnitude given by Caon et al. within the radial range of $1^{\prime}$ to 1'5. Bottom: Corresponding background values for the four profiles. radii. We excluded regions with bright stars, other galaxies, and obvious flat-field structure (chip 4, chip 5, and the upper part of chip 8). The inner $2^{\prime}$ are saturated.

The most difficult part in determining the galaxy luminosity profile is the correct determination of the sky background: a tiny $0.5 \%$ deviation in the sky level already has a profound effect on the galaxy light distribution as shown in Figure 14 for the $T 1$ profile. In Figure 14 (top) the luminosity profile is given for different sky values; these sky levels are indicated in a linear intensity scale in Figure 14 (bottom), where the $x$-axis is enlarged. Individual measurements are plotted as dots. If we would have reached the true sky background, profile $(b)$ would be appropriate and our resulting luminosity profile would look nearly identical to that of Caon et al. (1994). However, the measurements suggest that the galaxy light has not yet leveled out. On the other hand profile $(a)$ can be excluded, since the observed background is clearly lower than the one belonging to this profile. The profile by Schombert et al. can be reproduced (profile $[d]$ ) when using the lowest background in Figure 14 (bottom), but such a low background is almost certainly excluded since in this case the galaxy would have a radial extension of approximately $500 \mathrm{kpc}$, and hence it would be one of the largest galaxies known. As will be discussed below, profile (c), based on our preferred background, also resembles well that of the GCs, and thus we adopt it as our preferred profile. The difference with the $B$ profile of Caon et al. would still suggest a color that becomes redder with increasing distance, contrary to what we find (see below). Only profile (a) delivers a color gradient in the required sense, but this is not supported by our data.

From Figure 14 it is also apparent that the profile becomes rather diffuse for radii smaller than approximately $6^{\prime}$. The reason for this is the nonnegligible ellipticity of NGC 1399. The lower profile envelope belongs to the minor axis while the upper one belongs to the major axis. We determined an average decline only, but this poses no problem for the comparison with the cluster distribution, which has also been determined assuming a circular geometry. Moreover, the slope of a power law is not altered by averaging over the ellipticity. We nevertheless used this information to derive the radial dependence of the ellipticity, which is shown in the next section. The $C$ profile reaches the background level already at $13^{\prime}$ because of the shallower $C$ data, and we determine the background by averaging the "profile" at larger radii.

Between 2.5 and 9' (where the effects of the background uncertainty are still tolerable) we fitted power laws to the profile and obtained exponents of $-1.85 \pm 0.03$ for the $T 1$ profile and $-1.21 \pm 0.02$ for the $C$ profile, thus finding a clearly shallower $C$ profile. This holds for any sky background that deviates no more than $2 \%$ from our measured background, to which we assign an error of less than $1 \%$. The color gradient in $C-T 1$ is shown in Figure 20 (bottom) (see $\S 7.1$ ). The color gradient is also plotted as a smoothed curve, together with error limits estimated from the background uncertainties, in Figure 21.

We see that the color gradient might level out to a constant value of $C-T 1$ around 1.2 at $6^{\prime}$, which is approximately the same radius beyond which the blue and red GCs start to show indistinguishable surface density profiles. This might be evidence that the behavior of the GCS is indeed reflected in the field star population. However, the mean (linearly averaged) color of the clusters at this radius is 


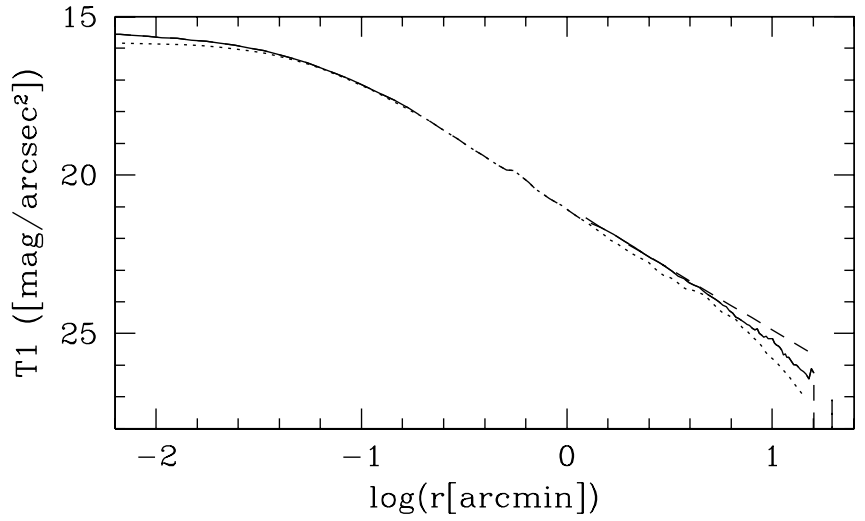

FIG. 15.-T1-luminosity profile of NGC 1399. We shifted all profiles (even if measured in a different passband) to our profile, the solid line at large radii. The profile of Caon et al. (1994) is the short-dashed curve, the one of Schombert et al. (1986) is the long-dashed curve, and the HST profile by Lauer et al. (1995) is the solid line at small radii.

$C-T 1=1.47$ and hence is considerably redder than the galaxy color.

\subsection{Covering the Whole Radial Range}

We are unable to determine the total magnitude of NGC 1399 because of the missing inner $2^{\prime}$. However, we can proceed by taking this data from the literature. In Figure 15 we plot the available luminosity profiles, which were shifted onto our $T 1$ magnitude scale by using our $T 1$ profile. We shifted the data of Caon et al. and Schombert et al. in the range between $1^{\prime}$ and 1.5. In the innermost region a $V$ profile based on $H S T$ observations has been published by Lauer et al. 1995, which we used out to $12^{\prime \prime}$. In the range between $12^{\prime \prime}$ and 1.5 , we used the $B$ profile of Caon et al. (1994). For larger radii, we used our profile. Color gradients in $B-R$ and $V-R$ in the inner region are very small and should not introduce significant errors (e.g., Idiart, Michard, \& de Freitas Pacheco 2002).

From this data we can construct a luminosity profile for NGC 1399 that covers 3 orders of magnitude in radius. In Table 4 we list this profile, together with the integrated $T 1$ magnitude at selected radii.

A good fit to the surface brightness profile of NGC 1399 is

$$
T 1(r)=2.125 \log \left[1+\left(\frac{r}{0.055}\right)^{2}\right]+15.75 .
$$

The deviation from the fit is shown in Figure 16, where we plot the difference between observed and fitted magnitude. Similar to the globular cluster density profile, the fit to the light profile using the whole radial range also results in a profile too steep at large radii (see Fig. 16). For these large radii, an exponent of 1.83 as derived above is more adequate. Integrating this profile, one obtains the total $R$ luminosity of NGC 1399, $M_{R}=-23.33$ (assuming a distance modulus of 31.4 and an absolute solar luminosity of $\left.M_{R \odot}=4.28\right)$.

If we adopt spherical geometry, one can derive the deprojected profile. Because we have the profile as an analytic expression, an easier way than to apply the deprojection formula is to adopt a power law of the above form, use the projection formula, and vary the parameters until the best fit is found.
TABLE 4

The Final Luminosity Profile of NGC 1399 Plotted in FIGURE 14

\begin{tabular}{|c|c|c|c|c|}
\hline $\begin{array}{c}\log r \\
(r \text { in } \operatorname{arcmin}) \\
(1)\end{array}$ & $\begin{array}{c}\mathrm{T} 1 \\
\left(\operatorname{arcsec}^{-2}\right) \\
(2)\end{array}$ & $\begin{array}{c}\text { Ellipticity } \\
\text { (3) }\end{array}$ & $\begin{array}{c}\mathrm{T} 1 \\
(\mathrm{mag}) \\
(4)\end{array}$ & $\begin{array}{c}C-T 1 \\
(5)\end{array}$ \\
\hline-3.436 & 15.39 & 0 & $\ldots$ & $\ldots$ \\
\hline$-2.436 \ldots \ldots \ldots \ldots$ & 15.48 & 0.13 & $\ldots$ & $\ldots$ \\
\hline$-2.115 \ldots \ldots \ldots$ & 15.59 & 0.13 & $\ldots$ & $\ldots$ \\
\hline$-1.757 \ldots \ldots \ldots \ldots$ & 15.78 & 0.062 & $\ldots$ & $\ldots$ \\
\hline$-1.405 \ldots \ldots \ldots \ldots$ & 16.19 & 0.085 & $\ldots$ & $\ldots$ \\
\hline$-1.052 \ldots \ldots \ldots$ & 16.99 & 0.12 & $\ldots$ & $\ldots$ \\
\hline$-1.015 \ldots \ldots \ldots \ldots$ & & & 11.38 & $\ldots$ \\
\hline-0.7095 & 18.14 & 0.13 & $\ldots$ & $\ldots$ \\
\hline$-0.4893 \ldots \ldots \ldots$ & 19.05 & 0.11 & $\ldots$ & $\ldots$ \\
\hline$-0.2939 \ldots \ldots \ldots$ & 19.84 & 0.11 & $\ldots$ & $\ldots$ \\
\hline$-0.1183 \ldots \ldots \ldots$ & 20.56 & 0.09 & $\ldots$ & $\ldots$ \\
\hline $0.0 \ldots \ldots \ldots \ldots \ldots \ldots$ & $\ldots$ & . & 9.43 & $\ldots$ \\
\hline $0.04123 \ldots \ldots \ldots$ & 21.27 & 0.07 & $\ldots$ & 1.71 \\
\hline $0.3299 \ldots \ldots \ldots \ldots$ & 22.43 & 0.09 & $\ldots$ & 1.64 \\
\hline $0.5136 \ldots \ldots \ldots \ldots$ & 23.18 & 0.11 & $\ldots$ & 1.55 \\
\hline $0.6422 \ldots \ldots \ldots \ldots$ & 23.69 & 0.20 & $\ldots$ & 1.41 \\
\hline $0.7413 \ldots \ldots \ldots \ldots$ & 24.14 & 0.20 & $\ldots$ & 1.36 \\
\hline $0.75 \ldots \ldots \ldots \ldots \ldots$ & $\ldots$ & $\ldots$ & 8.48 & $\ldots$ \\
\hline 0.822 & 24.63 & 0.20 & $\ldots$ & 1.13 \\
\hline $0.89 \ldots$ & 24.91 & 0.20 & $\ldots$ & 1.19 \\
\hline $0.9488 \ldots \ldots \ldots \ldots$ & 25.22 & 0.20 & $\ldots$ & 1.17 \\
\hline $1.001 \ldots \ldots \ldots \ldots \ldots$ & 25.31 & 0.20 & $\ldots$ & 1.29 \\
\hline $1.047 \ldots \ldots \ldots \ldots . . .$. & 25.69 & 0.20 & $\ldots$ & 1.03 \\
\hline $1.05 \ldots \ldots \ldots \ldots \ldots$ & $\ldots$ & $\ldots$ & 8.20 & $\ldots$ \\
\hline $1.089 \ldots \ldots \ldots \ldots$ & 26.03 & 0.20 & $\ldots$ & 1.05 \\
\hline $1.123 \ldots \ldots \ldots \ldots$ & 26.24 & 0.20 & $\ldots$ & $\ldots$ \\
\hline $1.191 \ldots \ldots \ldots \ldots$ & 26.21 & 0.20 & $\ldots$ & $\ldots$ \\
\hline $1.292 \ldots \ldots \ldots \ldots$ & 26.75 & 0.20 & $\ldots$ & $\ldots$ \\
\hline $1.292 \ldots \ldots \ldots \ldots$ & $\ldots$ & $\ldots$ & 8.08 & $\ldots$ \\
\hline
\end{tabular}

Note.-Col. (2): Surface brightness at the radius given in Col. (1); Col. (3): ellipticity; Col. (4): integrated magnitudes at selected radii; Col. (5): $C-T 1$ color.

Using this procedure, the best fit we obtain is

$$
L(T 1)\left(\frac{L_{\odot}}{1 \mathrm{pc}^{3}}\right)=101\left(1+\frac{r}{221 \mathrm{pc}}\right)^{-2.85} .
$$

We also determined the ellipticity of NGC 1399. However, we did not try to fit a position angle to the rather noisy data but estimated the position angle rather by eye inspection of

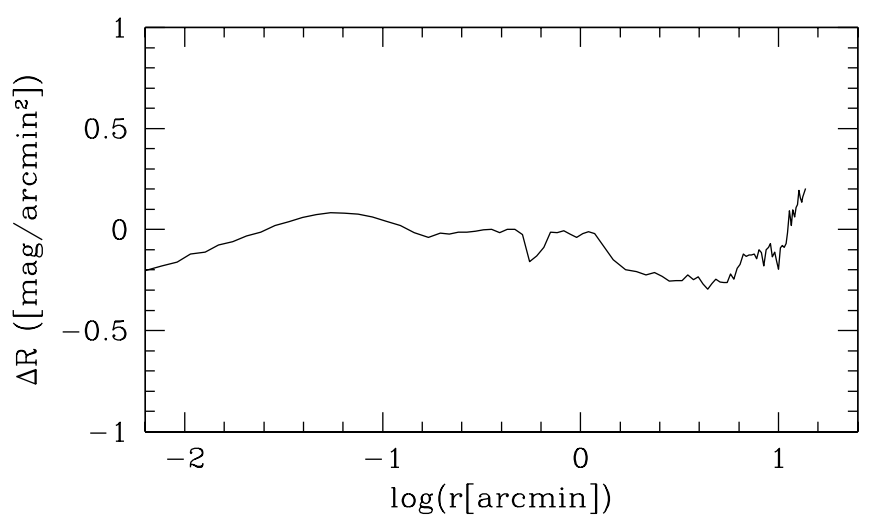

FIG. 16.-Difference between the observed and fitted galaxy light profile. Positive values mean fit values brighter than observed. 


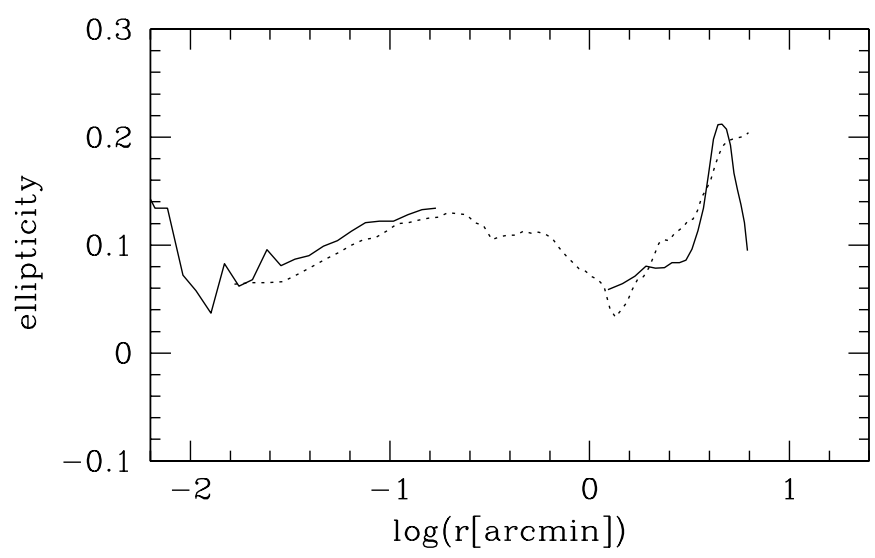

FIG. 17.- Radial dependence of the ellipticity of the galaxy $T 1$ light plotted in the outer region (solid lines) by using our MOSAIC data (using a fixed position angle at $90^{\circ}$ ) and in the inner part by using that of Lauer et al. (1995). The dashed line shows the ellipticity derived by Caon et al. (1994).

the image. We used a fixed position angle of $90^{\circ}$. The position angle variation from $110^{\circ}$ to $85^{\circ}$ seen by Caon et al. (1994) is not critical since it occurs between $30^{\prime \prime}$ and $1 ! 35$, while our profile starts at $2^{\prime}$. The resulting radial dependence of the ellipticity is shown in Figure 17, together with the values of Caon et al. The two measurements are in good agreement. The ellipticity of NGC 1399 is 0.1 between $2^{\prime}$ and $4^{\prime}$, then it rises to about 0.2 at $5^{\prime}$. The further decline at larger radii is rather uncertain, since at these large radii our azimuthal coverage is not homogeneous because of the exclusion of areas with bright stars, other galaxies, and obviously remaining flat-field structure.

In Figure 18 we compare the integrated light profile with aperture measurements from the literature. This figure shows that our determination agrees well with the literature values and that our data do not suggest any systematic zeropoint difference.

\section{SPECIFIC FREQUENCY}

The specific frequency is the number of globular clusters per unit luminosity scaled to a galaxy with an absolute luminosity of $M_{V}=-15 \mathrm{mag}\left[S_{V}=N \times 10^{0.4\left(M_{V}-15\right)}\right]$.

In Figure 11, the galaxy light profile is compared with the cluster density profile over nearly the whole observed radial

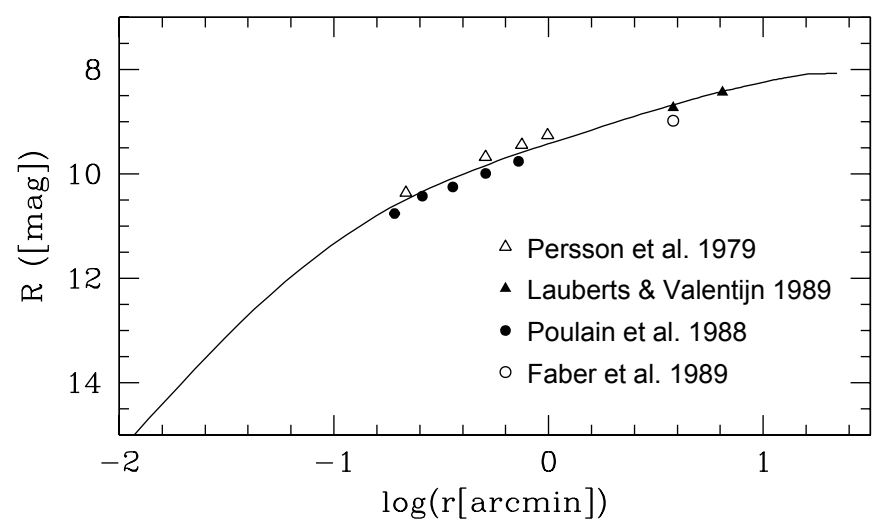

FIG. 18.- Integrated $R$ magnitude dependence on radius for our data and from various literature sources. For this purpose the $T 1$ measurements have been transformed into $R$ measurements. range. Determining the specific frequency means integrating over both curves. In the inner part the galaxy light is more concentrated than the globular clusters, which reflects the fact that the core radius of the GCS is about 18 times larger than that of the field population. This also means that the specific frequency is not radially constant. We shall proceed by first deriving a global value and then studying the radial dependence of the specific frequency for both red and blue clusters.

\subsection{Global Specific Frequency}

To derive the total number of clusters we have to account for the incompleteness with respect to the globular cluster luminosity function. In $\S 4$ we determined this to be $35 \% \pm 5 \%$.

Within the radial range from $2^{\prime}$ to $17^{\prime}$ (excluding a radius of $2^{\prime}$ around NGC 1404), we find $2600 \pm 50$ cluster candidates. Within the central $2^{\prime}$ Forbes et al. (1998) found 700 clusters. From our background field we know that a cluster candidate sample brighter than $T 1=23.5$ has a contamination of $0.48 \pm 0.02$ objects $\mathrm{arcmin}^{-2}$. Accounting for this contamination and incompleteness, we find a total number of $6100 \pm 770$ clusters within $83 \mathrm{kpc}\left(15^{\prime}\right)$.

Alternatively, we integrated the density profile derived in $\S 7$ and obtained $6800 \pm 950$ clusters within $83 \mathrm{kpc}$. Within $10^{\prime}$, this results in 5793 clusters. Averaging the results of both determinations we find the total number of clusters within $15^{\prime}$ to be $6450 \pm 700$. Our obtained number agrees well with that found for the cluster content within $10^{\prime}$. Ostrov et al. (1998), Forbes et al. (1998), and Kissler-Patig et al. (1997) all quote $\approx 5700$ clusters within $10^{\prime}$. In the inner part we have an excellent agreement with older studies, too: within 1.5 and 2.5 we determine a total number of $734 \pm 114$ clusters, while Ostrov et al. found $746 \pm 80$, and within 2.5 and $4^{\prime}$ we have $921 \pm 140$ whereas Ostrov et al. give $967 \pm 100$.

With this total number of clusters, we find a global specific frequency of $S_{R}=3.2 \pm 0.7$ by using a total galaxy magnitude of $T 1=8.08$ (Table 5) and $m-M=31.4 \pm 0.2$. Since we cannot measure the galaxy light in the $V$ band, we have to convert $T 1$ into $V$ magnitudes to be able to compare our value with the literature. Using a color of $V-R=0.55$ (Mackie, Visvanathan, \& Carter 1990) we find a specific frequency of $S_{V}=5.1 \pm 1.2$ within $15^{\prime}$.

Forbes et al. (1998) quoted a specific frequency of $11.5 \pm 1$. They used an absolute magnitude of $M_{V}=$ -21.74 (taken from Faber et al. 1989), while we would use $T 1=8.25$, which translates to $M_{V}=-22.59$ (see above) for the total luminosity (within $10^{\prime}$ ). Part of the difference comes from the different distance modulus adopted by Forbes et al. $(m-M=31.2)$. However, the major discrepancy is in the apparent magnitude: Faber et al. give the $B_{T}$, magnitude, which is defined as the magnitude within the $25 \mathrm{mag}$ $\operatorname{arcsec}^{-2}$ isophote. Using a $B-R=1.8$ value, we find that 25 mag $\operatorname{arcsec}^{-2}$ corresponds to a radius of 3!.8. Within this radius we measure an approximate $B$ luminosity (again assuming no $B-R$ color gradient) of $10.4 \mathrm{mag}$, which is in reasonable agreement, considering all uncertainties to the Faber et al. value of $B=10.55$. The same argument can also be applied to explain the high specific frequencies determined by Kissler-Patig et al. (1997), Wagner et al. (1991), and Bridges et al. (1991). This shows that one of the most important caveats in deriving the specific frequency is that 
TABLE 5

Local Specific Frequency

\begin{tabular}{|c|c|c|c|}
\hline $\begin{array}{c}\log r \\
(r \text { in arcmin) } \\
(1)\end{array}$ & $\begin{array}{c}S_{R}(\text { all }) \\
(2)\end{array}$ & $\begin{array}{c}S_{R}(\mathrm{red}) \\
\quad \text { (3) }\end{array}$ & $\begin{array}{c}S_{R} \text { (blue) } \\
\text { (4) }\end{array}$ \\
\hline $0.26 \ldots \ldots \ldots \ldots \ldots$ & $4.3 \pm 0.4$ & $2.6 \pm 0.32$ & $1.7 \pm 0.3$ \\
\hline $0.43 \ldots \ldots$ & $5.8 \pm 0.7$ & $3.3 \pm 0.5$ & $2.5 \pm 0.5$ \\
\hline $0.56 \ldots \ldots \ldots \ldots \ldots$ & $6.9 \pm 1.0$ & $3.7 \pm 0.7$ & $3.2 \pm 0.7$ \\
\hline $0.65 \ldots \ldots \ldots \ldots \ldots$ & $7.0 \pm 1.2$ & $3.3 \pm 0.8$ & $3.7 \pm 0.9$ \\
\hline $0.73 \ldots$ & $8.2 \pm 1.5$ & $3.7 \pm 1.0$ & $4.5 \pm 1.1$ \\
\hline $0.80 \ldots \ldots \ldots$ & $9.1 \pm 1.9$ & $4.0 \pm 1.3$ & $5.1 \pm 1.4$ \\
\hline $0.86 \ldots \ldots \ldots \ldots \ldots$ & $9.8 \pm 2.3$ & $4.7 \pm 1.6$ & $5.1 \pm 1.6$ \\
\hline 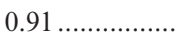 & $9.3 \pm 2.4$ & $3.4 \pm 1.5$ & $6.0 \pm 1.9$ \\
\hline $0.95 \ldots \ldots \ldots \ldots \ldots . . .$. & $7.5 \pm 2.4$ & $3.3 \pm 1.6$ & $4.1 \pm 1.8$ \\
\hline 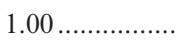 & $8.1 \pm 2.7$ & $2.8 \pm 1.6$ & $5.3 \pm 2.2$ \\
\hline 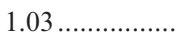 & $6.4 \pm 2.7$ & $3.3 \pm 1.9$ & $3.1 \pm 1.9$ \\
\hline 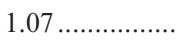 & $10.8 \pm 3.9$ & $4.9 \pm 2.7$ & $5.9 \pm 2.9$ \\
\hline $1.10 \ldots \ldots \ldots \ldots \ldots \ldots$ & $14.2 \pm 5.0$ & $4.9 \pm 2.9$ & $9.2 \pm 4.0$ \\
\hline 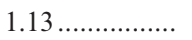 & $12.6 \pm 5.0$ & $5.5 \pm 3.3$ & $7.1 \pm 3.7$ \\
\hline $1.16 \ldots \ldots \ldots \ldots \ldots . . . .$. & $11.8 \pm 5.4$ & $5.3 \pm 3.6$ & $6.5 \pm 4.0$ \\
\hline $1.20 \ldots \ldots \ldots \ldots \ldots$ & $18.3 \pm 9.0$ & $9.9 \pm 6.6$ & $8.4 \pm 6.1$ \\
\hline
\end{tabular}

Note.-Col. (2): local specific frequency for the whole sample; Col. (3): the red subpopulation; Col. (4): the blue sample. The errors include the error of the luminosity profile and Poisson counting statistics.

the clusters and the galaxy light has to be measured within the same area, a fact that has already been pointed out by Ostrov et al. (1998) for the case of NGC 1399.

\subsection{Local Specific Frequency}

Because of the existence of subpopulations in the GCS, it is useful to define a local specific frequency in which the number of GCs is normalized to the brightness of the area in which they are counted. The local specific frequency of the red, the blue, and the total cluster sample for different radial bins is given in Table 5 and displayed in Figure 19. Since the galaxy light profile in the $R$ band matches well the radial distribution of the red cluster population within $11^{\prime}$, its local specific frequency remains nearly constant around $S_{R}=3$. Outside $11^{\prime}$ small background uncertainties make a definite statement on the surface brightness profile difficult. However, using a reasonable background it is easy to find a galaxy profile that also follows the red clusters at these large

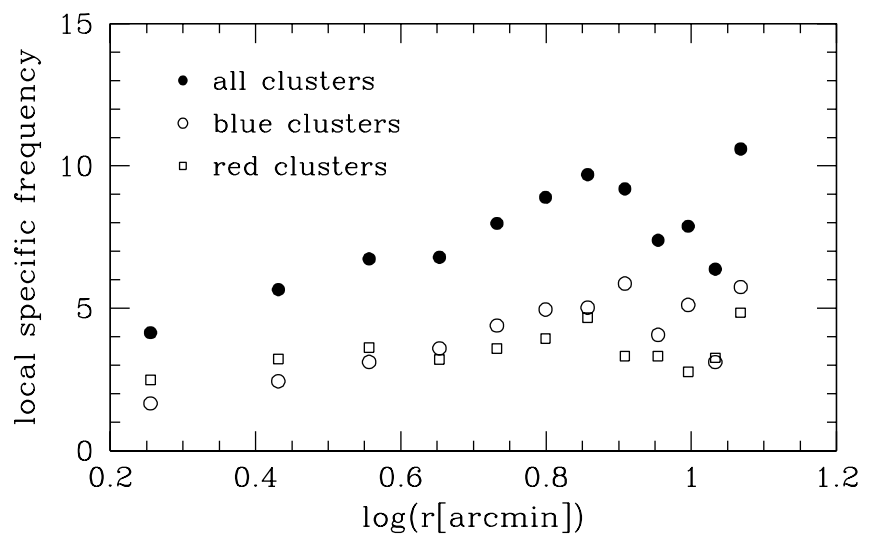

FIG. 19.-Radial local specific frequency plotted for the whole cluster sample and for the metal-rich and metal-poor subsamples. radii. The blue cluster system, on the other hand, within $11^{\prime}$ shows a slightly shallower profile and therefore its specific frequency with respect to the $R$-band luminosity increases outward in proportion to $r^{0.8 \pm 0.2}$. However, the behavior at larger radii is uncertain as well.

\section{DISCUSSION}

\subsection{Cluster Color Distribution}

In general, there is little doubt that the color of a $\mathrm{GC}$ indicates its metallicity in an old elliptical galaxy such as NGC 1399 (however, we cannot exclude that younger clusters are mixed in; Forbes et al. 2001). The GC color distribution has been the starting point for many investigations regarding the substructure of GCSs of elliptical galaxies. It has been found by many authors (e.g., Kundu \& Whitmore 2001a; Forbes \& Forte 2001; Larsen et al. 2001; Gebhardt \& Kissler-Patig 1999; see also the corresponding section in the book of Ashman \& Zepf 1998) that GCSs often exhibit two peaks in the color distribution, labeled "bimodality." If bimodality is not obvious then frequently at least the existence of two peaks is statistically better supported than a unimodal color distribution. Usually a linear transformation between color and metallicity without accounting for an intrinsic scatter around the mean color metallicity is applied (e.g., Harris et al. 2000; Kissler-Patig et al. 1997; Harris et al 1991). This means that a bimodal color distribution translates into a bimodal metallicity distribution that is assigned to two different populations. Because bimodal color distributions of clusters in elliptical galaxies have been predicted by Ashman \& Zepf (1992) in the context of a simple merger scenario, color bimodality has been interpreted supporting the formation of GCSs of elliptical host galaxies in merger events. However, despite the fact that a merger can produce a bimodal color distribution it is not clear whether a bimodal distribution necessarily implies a merger scenario; e.g., even in a monolithic collapse scenario a bimodal metallicity distribution (of stars) can be obtained: Samland, Hensler, \& Theis (1997) simulated the formation of a disk galaxy starting with a coherent gas cloud. They found that disk, halo, and bulge stars have different metallicity distributions (their Fig. 7). If one plots their Figure 7 in logarithmic metallicity bins a bi- or even multimodal metallicity distribution becomes apparent. They note "that a bimodal star formation or a pre-enrichment of the protogalactic cloud is not required by our model. The metallicity distributions for the different galactic regions result purely from the local star formation history, the large-scale gas flows, and the phase transitions by condensation and evaporation."

We also would like to call attention to the fact that the C-T1 color-metallicity relation is nonlinear, flattening out in the metal-poor regime (Harris \& Harris 2002). In conjunction with an intrinsic spread in the color-metallicity relation due to second parameters and photometric errors, this leads to the circumstance that starting from the bluest colors equidistant color intervals are projected onto progressively larger metallicity intervals, which decrease once the linear regime is reached. What this effect means for the interpretation, in particular of the blue peak, is certainly worth investigating but beyond the scope of our paper.

The question of whether the color distribution of a GCS depends on the host galaxy's properties is still unclear. Regarding the Washington system only a few GCSs have 
been studied: Geisler et al. (1996) investigated the GCS of NGC 4472 and obtained $C-T 1 \approx 1.3$ and $C-T 1 \approx 1.8$ for the colors of the blue and read peak, respectively. The blue peak of NGC 1427, a low-luminosity elliptical galaxy, also agrees with the blue peak of the NGC 1399 clusters $(C-T 1 \approx 1.4$; Forte et al. 2001). M87 also has a GCS with a bimodal color distribution that peaks at $C-T 1 \approx 1.25$, while the position of the red peak remains uncertain in the color histogram shown by Côté et al. 2001, which is based on still unpublished photometry. Two more GCSs for which Washington photometry have been obtained are those of NGC 3311 (Secker et al. 1995) and NGC 3923 (Zepf, Ashman, \& Geisler 1995). Both galaxies (observed during the same run) show abnormally red color distributions. However, Brodie, Larsen, \& Kissler-Patig 2000 performed $V-I$ photometry with HST in NGC 3311 and found a normal bimodal distribution with peaks at the expected colors. Since the Washington run was not photometric, one might conjecture that both NGC 3311 and NGC 3923 were subject to calibration uncertainties. Together with our results on NGC 1399 we conclude that at least the blue peaks have very similar colors in those GCSs of elliptical galaxies investigated so far with Washington photometry.

\subsection{Interpreting the Radial Cluster Distribution}

In Paper II, we will show that the blue and red clusters within $7^{\prime}$ are also kinematically distinct: The red clusters have a velocity dispersion of $274 \pm 15 \mathrm{~km} \mathrm{~s}^{-1}$, while the blue clusters have $310 \pm 10 \mathrm{~km} \mathrm{~s}^{-1}$. This larger dispersion can be well accounted for in the framework of a spherically dynamical model because of the different surface density slopes. In other words, a higher (lower) dispersion is dynamically equivalent to a shallower (steeper) surface density profile in an isotropic equilibrium situation.

A distinction of two subpopulations on the basis of the color histogram alone bears a high degree of uncertainty. However, in light of the correlation between metallicity and density profile kinematics, we find it justified to talk about two populations out to a radius of about $7 '$. However, that does not necessarily imply the existence of two distinct populations, in the sense of different formation epochs or formation mechanisms. It may well be that there is a continuum of properties, over which our color sampling is simply taking an average. At larger galactocentric radii, the distinction between subpopulations becomes much less pronounced. The density profiles of blue and red clusters are more or less the same and the color distribution indicates a broad range of metallicities, even if two Gaussians are still a better representation that one Gaussian, but as we said above we do not assign too much physical significance to that.

Despite the large field used we have not yet reached the background. The total extension of the GCS of NGC 1399 is hence not yet known. However, for GCs with a very large radii the question remains whether such clusters should be considered to belong to NGC 1399 or to be an "intergalactic GC population" (White 1987a; West et al. 1995). Bassino et al. (2003) used Washington observations around dwarf elliptical galaxies in the Fornax clusters to search for GCs at large radii. They found a GC candidate surface density of $0.25 \pm 0.08 \mathrm{arcmin}^{-2}$ and $0.12 \pm 0.06 \mathrm{arcmin}^{-2}$ at a radial distance of $40^{\prime}$ and $2^{\circ}$ from NGC 1399, respectively. For comparison, using their limiting magnitude of
$R=22$ mag, we find a surface density of $0.3 \pm 0.1 \mathrm{arcmin}^{-2}$ at a radial distance of $16^{\prime}$. Extrapolating our derived radial profile we would expect a surface density of $\approx 0.14 \mathrm{arcmin}^{-2}$ at $40^{\prime}$ and of $\approx 0.02 \operatorname{arcmin}^{-2}$ at $2^{\circ}$. In particular at the larger radius we would expect a smaller number of GCs. This could be an indication that a population of "intergalactic clusters" exists, as proposed by Bassino et al. (2003).

\subsection{Comparison of the NGC 1399 Light Profile with That of a "Normal" Elliptical Galaxy}

The luminosity profiles of many " normal" elliptical galaxies follow a $r^{1 / 4}$ de Vaucouleurs law. Here we have the opportunity to differentially compare NGC 1399 with such a "normal" galaxy, NGC 1404. This difference is illustrated in Figure 20, where the profiles of NGC 1399 and of NGC 1404 are compared. We derived the luminosity profile of NGC 1404 in $C$ and $T 1$ in the same way as for NGC 1399. Care has been taken to account for the extended halo of NGC 1399, which fully envelops NGC 1404 in projection. We subtracted its contribution, i.e., the final luminosity profile, from each pixel. For the sky background the same value as for NGC 1399 has been used. It can be seen that the light profile of NGC 1404, in contrast to NGC 1399, does not exhibit a uniform power law but has an exponent of about -2 for radii smaller than 1.8 and -2.9 for larger radii, i.e., almost -4 in deprojection.

The deviation from a $r^{1 / 4}$ de Vaucouleurs law is not unusual for galaxies brighter than $M_{V}=-21$. The light profile shapes of brighter galaxies become progressively
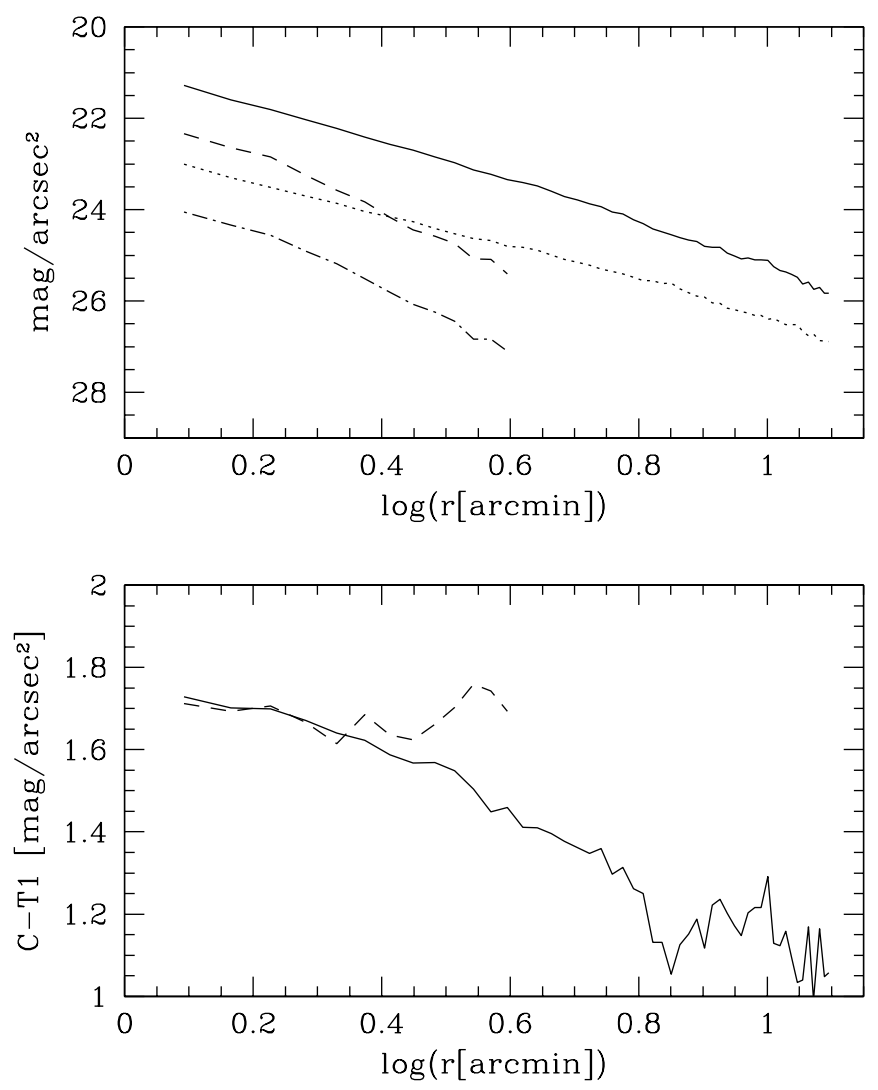

FIG. 20.-Top, Luminosity profile in $T 1$ (solid line) and $C$ (dotted line) of NGC 1399 compared with the T1 (dashed line) and C (dash-dotted line) surface luminosity of NGC 1404; bottom, radial color plotted for NGC 1399 (solid line) and NGC 1404 (dashed line). 
more concave when plotted against $r^{1 / 4}$ (Kormendy \& Djorgovski 1989). These authors define a cD galaxy as a light profile that exhibits an inflection point independent of the way it is plotted. In this sense, NGC 1399 would not be a $\mathrm{cD}$ galaxy.

Whatever the exact definition of a cD galaxy may be, it is useful for further insight to think about the physical reason of a de Vaucouleurs profile. Many "normal" elliptical galaxies can be well described by a Jaffe (1987) profile, which in projection resembles the de Vaucouleurs law, and which in space in the outer regions is characterized by a transition from an $r^{-3}$ to an $r^{-4}$ power law. Jaffe (1987) and White (1987b) have shown that an $r^{-4}$ power law emerges if the energy distribution function has a sharp break at the escape energy, given a nearly Keplerian potential.

NGC 1399 has a massive dark halo resembling the potential of an isothermal sphere out to a distance of at least 40 $\mathrm{kpc}$, shown by the dynamics of the globular clusters (Paper II). The temperature of the X-ray gas outside this radius remains isothermal (Jones, Stern, \& Forman 1997). Therefore, even if the exact shape of the potential is not known, we can at least conclude that the Keplerian regime is not reached out to the very faint surface brightnesses where our photometry becomes unreliable. Thus it is very tempting to relate the persistence of the $r^{-3}$ profile to the dark halo of NGC 1399.

We therefore understand the "halo" of NGC 1399, which morphologically appears as a deviation from a de Vaucouleurs profile, not as an additional component but as the natural continuation of the galaxy profile in the isothermal potential of the dark matter halo. Thus the term " $\mathrm{cD}$ halo" might be misleading since it implies an additional stellar component.

Finally, we do not detect a color gradient for NGC 1404. This shows that the population composition of NGC 1404 is radially more homogeneous than that of NGC 1399. Additionally, this also demonstrates that the observed color gradient in NGC 1399 is not an artifact of an incorrect background subtraction.

\subsection{Disentangling the Stellar Populations}

Superior to the approach that relates the two observed red and blue cluster subpopulations to the integral galaxy light would be the approach that compares clusters and the respective field populations that formed during the same star formation event. This is not possible in a direct way. However, we can follow a more indirect approach, in which we model the galaxy $C$ and $R$ light profile by using some simplifying assumptions. We assume the field population to be a superposition of only two populations, one corresponding to the blue clusters and one to the red clusters. These two field populations shall have the mean color of their corresponding cluster populations. The relation between the number of clusters and the luminosity of their corresponding field population will be called the intrinsic specific frequency (ISF) and is measured with respect to the $T 1$ filter.

It is not possible to fit both the color and the luminosity profile if the two cluster populations would have the same constant ISF because of their different surface density profiles. In addition, to reproduce the color gradient requires allowing for a change of the properties of the red populations by either a radial color or radial local specific

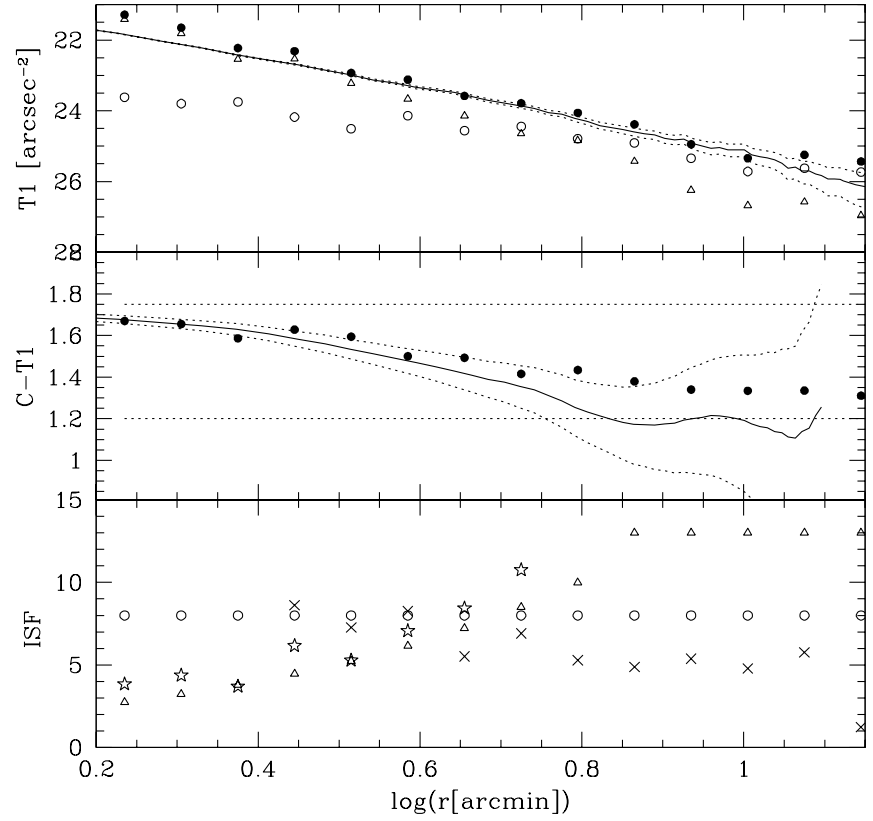

Fig. 21.-Simple model to fit the galaxy light profile measured in the $C$ and $R$ bands. Top: Galaxy light shown as solid lines accompanied by estimated errors due to background variations. The filled circles show the model light distribution, that is, a composite of the contribution of the red (triangles) and blue (open circles) populations. The two horizontal lines indicate the color used as mean color for the two populations. Middle: Color profile plotted as a solid line, together with the estimated errors and compared with the model color distribution (filled circles). The two horizontal lines indicate the value used as the mean color for each population. Bottom: ISFs that define the model for the blue (open circles) and red population (open triangles) and the ISF for the blue (crosses) and red (diamonds) populations when it is directly calculated.

frequency dependence. We limit ourselves to consider a changing ISF for the red population, which might be the strongest assumption we make. Otherwise the problem would not be well constrained and many solutions could be found. We used the linear approximation ISF $\propto r$. A shallower variation, e.g., ISF $\propto \log r$, results in too shallow a color gradient. With a much steeper dependence, e.g., ISF $\propto r^{2}$, the model luminosity profile shows a considerable dip at the point where the light of the blue population begins to dominate the total light, which is also incompatible with the observations. A model that fits the data reasonably well is depicted in Figure 21. A characteristic of any satisfactory model is that the ISF of the blue cluster population is much higher than that of the red one at smaller radii.

It is also possible to use the color and luminosity profile together with the density profiles of red and blue clusters to derive the ISF for both cluster samples directly. However, the disadvantage of this approach is that it is much more strongly influenced by noise. Nevertheless, it may serve as a consistency test, and we include the results of this calculation in Figure 21. This illustrates that the two methods agree well considering the uncertainties.

\subsection{From Light Distribution to Mass Distribution}

We note that the color gradient in NGC 1399 indicates a radial change in the stellar $M / L$ profile. If the outer regions are more metal-poor the $M / L$ value decreases toward larger radii. If one wants to use the luminosity profile to infer the mass profile, one has to account for this effect. For an estimation, we assume two populations with the same age, 
12 Gyr, and different mean metallicities: a metallicity of -0.4 dex (representing the inner part in accordance with the galaxy's color) and -1.4 dex (representing the outermost parts). Worthey's interpolation engine then gives for the respective $M / L$ values in $R$ (Miller-Scalo IMF) 1.45 $(-0.4 \mathrm{dex})$ and $1.23(-1.4 \mathrm{dex})$. This means that the associated mass profile becomes somewhat steeper than what would be derived from the luminosity profile using a constant $M / L$. Thus an increase of the power-law exponent from -1.83 to about -2.2 is expected.

\section{SUMMARY AND CONCLUSIONS}

We investigated the GCS of NGC 1399 in the Washington $C$ and Kron $R$ bands with the CTIO $4 \mathrm{~m}$ MOSAIC camera. The imaged area is $36^{\prime} \times 36^{\prime}$, thus among the largest concerning GCS investigations.

The main findings of this study are

The relatively modest specific frequency of $5.1 \pm 1.2$ of the GCS of NGC 1399, already noted by Ostrov et al. (1998) is confirmed. The local specific frequency increases with radius and is higher for the blue than for the red clusters.

The color distribution shows a very pronounced bimodal appearance. However, we argue that the blue peak might be artificially caused by the nonlinear color-metallicity relation in conjunction with the color scatter at a given metallicity.

The surface density profile is shallower for the blue clusters than for the red clusters within 8 arcmin. Outside a radius of $8^{\prime}$, both surface density profiles are not distinguishable.

The galaxy light shows a color gradient resembling the change of the ratio of the surface density profiles of blue and red clusters. The galaxy light can be modeled with the simple assumption of only two populations, each traced by a cluster population of the same mean color. A model consistent with the data has a constant ISF for the blue clusters and a radially increasing ISF for the red clusters.

The brightest clusters do not show the pronounced bimodal color distribution of the fainter ones and their radial distribution is at least as concentrated as that of the red GCs.

NGC 1399 was one of the "classic" high specific frequency galaxies. It would be very interesting to perform new studies of the similar cases M87 and NGC 3311 to see whether their high specific frequencies still withstand closer scrutiny. If not, central galaxies in galaxy clusters are perhaps not the places of extraordinarily high efficiency of globular cluster formation that they have been taken to be.

The GC color distribution, in particular bimodality, alone is not very restrictive for galaxy formation scenarios. Various models have been published that explain the observed bimodality/metallicity distribution, e.g., Ashman \& Zepf (1992), Forbes, Brodie, \& Grillmair (1997), Côté et al. (2001), and Beasley et al. (2002). It is believed that bimodal color distributions can be used as an argument against monolithic collapse scenarios, for example, Rhode \& Zepf (2001). However, doubts remain: Samland et al. (1997) showed that a monolithic collapse scenario also results in populations that have different metallicity distributions.

Since the color distribution alone is not sufficient to constrain formation scenarios it is necessary to use further information. The close relation of the color gradient of NGC 1399 to the mean color of the GCS might be seen as evidence in favor of a strongly dissipative period during which the globular clusters and the stellar body of the galaxy were formed together. However, a counterargument is suggested by the radially increasing local specific frequency of the red clusters. During a dissipative collapse one would expect the star formation rate to be highest where the density is highest, i.e., in the central regions. Accordingly, also the efficiency of globular cluster formation should decrease radially outward if the star formation rate is a determining parameter (Larsen \& Richtler 2000).

Additionally, one has to take into account the kinematic and dynamical properties of the GCS. These are the following (Paper II): Blue and red clusters show different velocity dispersions in accordance with their different surface density profiles. The orbit distribution is nearly isotropic. Only the outer blue clusters (if any) show signatures of rotation. The overall mass distribution resembles an isothermal sphere.

Thus some sort of relaxation mechanism must have been active in the early times of NGC 1399, which is difficult to find in a purely dissipative collapse but naturally provided by a phase of violent relaxation during a merger. The merger components (probably more than only two) could have been gas-rich, unevolved metal-poor galaxies where cluster formation was efficient during the starburst preceding the merger itself. In addition to the (presumably mainly metal-poor) cluster populations of the merger components, the bulk of clusters, both metal-rich and metal-poor, could have been formed during this period. After the merging took place these clusters have roughly the same spatial distribution. However, after the merger remnant has reached its equilibrium, cluster formation continued in the inner regions, albeit with a decreased efficiency, forming the metal-rich part of the inner red cluster population.

Based on this idea we can estimate the number of additional red globular clusters. For this we assume that we have one population of metal-rich clusters that have the same density profile as the blue ones (albeit with a different normalization taken from the red clusters at radii larger than $8^{\prime}$ ). Then we require $\approx 700$ additional red clusters to explain the difference between the two profiles.

We acknowledge the help of M. Shara, D. Zurek, and E. Grebel, who obtained some images that were used in this investigation. B. D. gratefully acknowledges financial support of the Alexander-von-Humboldt Foundation via a Feodor Lynen Stipendium. D. G. and W. P. G. gratefully acknowledge support from the Chilean Center for Astrophysics FONDAP 15010003.
Ashman, K. M., Conti, A., \& Zepf, S. E. 1995, AJ, 110, 1164

Ashman, K. M., \& Zepf, S. E. 1992, ApJ, 384, 50

_. 1998, Globular Cluster Systems (Cambridge Astrophys. Ser.) (Cambridge: Cambridge Univ. Press)

Bassino, L. P., Cellone, S. A., Forte, J. C., \& Dirsch, B. 2003, A\&A, in press

Beasley, M. A., Baugh, C. M., Ducan, A. F., Sharples, R. M., \& Frenck, C. S. 2002, MNRAS, 333, 383

\section{REFERENCES}

Bekki, K., Forbes, D. A., Beasley, M. A., \& Couch, W. J. 2002, MNRAS, 335, 1176

Bertin, E., \& Arnouts, S. 1996, A\&AS, 117, 393

Bridges, T. J., Hanes, D. A., \& Harris, W. E. 1991, AJ, 101, 469

Brodie, J. P., Larsen, S. S., \& Kissler-Patig, M. 2000, ApJ, 543, L19

Caon, N., Capaccioli, M., \& D’Onofrio, M. 1994, A\&AS, 106, 199

Côté, P., et al. 2001, ApJ, 559, 828

Dawe, J. A., \& Dickens, R. J. 1976, Nature, 263, 395 
Elmegreen, B. G. 1999, Ap\&SS, 269, 469

Faber, S. M., Wegner, G., Burstein, D., Davies, R. L., Dressler, A., Lynden-Bell, D., \& Terlevich, R. J. 1989, ApJS, 69, 763

Forbes, D. A., Beasley, M. A., Brodie, J. P., \& Kissler-Patig, M. 2001, ApJ, 563, L143

Forbes, D. A., Brodie, J. P., \& Grillmair, C. J. 1997, AJ, 113, 1652

Forbes, D. A., \& Forte, J. C. 2001, MNRAS, 322, 257

Forbes, D. A., Grillmair, C. J., Williger, G. M., Elson, R. A. W., \& Brodie, J. P. 1998, MNRAS, 293, 325

Forte, J. C., Geisler, D., Ostrov, P. G., Piatti, A., \& Gieren, W. 2001, AJ, 121,1992

Gebhardt, K., \& Kissler-Patig, M. 1999, AJ, 118, 1526

Geisler, D. 1996, AJ, 111, 480

Geisler, D., \& Forte, J. C. 1990, ApJ, 350, L5

Geisler, D., Lee, M. G., \& Kim, E. 1996, AJ, 111, 1529

Grillmair, C. J., Forbes, D. A., Brodie, J. P., \& Elson, R. A. W. 1999, AJ, 117,167

Hanes, D. A., \& Harris, W. E. 1986, ApJ, 309, 564

Harris, H. C., \& Canterna, R. 1977, AJ, 82, 798

Harris, W. E. 2001, in Star Clusters, Saas-Fee Advanced Course 28 Lecture

Notes, ed. L. Labhardt \& B. Binggeli (Berlin: Springer), 223

Harris, W. E., Allwright, J. W. B., Pritchet, C. J., \& van den Bergh, S. 1991, ApJS, 76, 115

Harris, W. E., \& Hanes, D. A. 1987a, AJ, 93, 1368

Harris, W. E., \& Harris, G. L. H. 2002, AJ, 123, 3108

Harris, W. E., Kavelaars, J. J., Hanes, D. A., Hesser, J. E., \& Pritchet, C. J. 2000, ApJ, 533, 137

Harris, W. E., \& van den Bergh, S. 1981, AJ, 86, 1627

Idiart, T. P., Michard, R., \& de Freitas Pacheco, J. A. 2002, A\&A, 383, 30

Jaffe, W. 1987, in IAU Symp. 127, Structure and Dynamics of Elliptical

Galaxies, ed. T. de Zeeuw \& D. Reidel (Dordrecht: IAU), 511

Jones, C., Stern, C., \& Forman, W. 1997, ApJ, 482, 143

Killeen, N. E. B., \& Bicknell, G. V. 1988, ApJ, 325, 165

Kissler, M., Richtler, T., Held, E., Grebel, E. K., Wagner, S. J., \& Capaccioli, M. 1994, A\&A, 287, 463

Kissler-Patig, M., Kohle, S., Hilker, M., Richtler, T., Infante, L., \& Quintana, H. 1997, A\&A, 319, 470

Kormendy, J., \& Djorgovski, S. 1989, ARA\&A, 27, 235
Kundu, A., \& Whitmore, B. C. 2001a, AJ, 121, 2950

2001b, AJ, 122, 1251

Larsen, S. S., Brodie, J. P., Huchra, J. P., Forbes, D. A., \& Grillmair, C. J. 2001, AJ, 121, 2974

Larsen, S. S., \& Richtler, R. 1999, A\&A, 345, 59 2000, A\&A, 354, 836

Lauer, T. R., et al. 1995, AJ, 110, 2622

Liu, M. C., Graham, J. R., \& Charlot, S. 2002, ApJ, 564, 216

Mackie, G., Visvanathan, N., \& Carter, D. 1990, ApJS, 73, 637

McLaughlin, D. E. 1999, AJ, 117, 2398

Merritt, D., \& Tremblay, B. 1994, AJ, 108, 514

Ostrov, P. G., Forte, J. C., \& Geisler, D. 1998, AJ, 116, 2854

Ostrov, P. G., Geisler, D., \& Forte, J. C. 1993, AJ, 105, 1762

Rhode, K. L., \& Zepf, S. E. 2001, AJ, 121, 210

Richtler, T., et al. 2002a, AJ, submitted (Paper II)

Richtler, T., et al. 2002b, in IAU Symp. 207, Extragalactic Star Clusters, ed. D. Geisler, E. K. Grebel, \& D. Minniti (San Francisco: ASP), 263

Richtler, T., Drenkhahn, G., Gómez, M., \& Seggewiss, W. 2000, in From Extrasolar Planets to Cosmology: The VLT Opening Symposium, ed.

J. Bergeron \& A. Renzini (Berlin: Springer), 259

Samland, M., Hensler, G., \& Theis, Ch. 1997, ApJ, 476, 544

Schlegel, D., Finkbeiner, D., \& Davis, M. 1998, ApJ, 500, 525

Schombert, J. M. 1986, ApJS, 60, 603

Secker, J., Geisler, D., Mc Laughlin, D. E., \& Harris, W. E. 1995, AJ, 109, 1019

Tonry, J. L., Dressler, A. D., Blakeslee, J. P., Ajhar, E. A., Flecher, A. B., Luppino, G. A., Metzger, M. R., \& Moore, C. B. 2001, ApJ, 546, 681

van den Bergh, S. 2000, PASP, 112, 932

Wagner, S., Richtler, T., \& Hopp, U. 1991, A\&A, 241, 399

West, M. J., Côté, P., Jones, C., Forman, W., \& Marzke, R. O. 1995, ApJ, 453, L77

White, R. E., III. 1987a, MNRAS, 227, 185

White, S. D. M. 1987b, in IAU Symp. 127, Structure and Dynamics of Elliptical Galaxies, ed. T. de Zeeuw \& D. Reidel (Dordrecht: IAU), 263

Whitmore, B. C., \& Schweizer, F. 1995, AJ, 109, 960

Worthey, G. 1994, ApJS, 95, 107

Zepf, S. E., \& Ashman, K. M. 1993, MNRAS, 264, 611

Zepf, S. E., Ashman, K. M., \& Geisler, D. 1995, ApJ, 443, 570 